ESAIM: COCV 20 (2014) 190-221

DOI: $10.1051 / \mathrm{cocv} / 2013061$
ESAIM: Control, Optimisation and Calculus of Variations

www.esaim-cocv.org

\title{
VARIATIONAL APPROXIMATION OF A FUNCTIONAL OF MUMFORD-SHAH TYPE IN CODIMENSION HIGHER THAN ONE
}

\author{
FRANCESCO GHIRALDIN ${ }^{1}$
}

\begin{abstract}
In this paper we consider a new kind of Mumford-Shah functional $E(u, \Omega)$ for maps $u: \mathbb{R}^{m} \rightarrow \mathbb{R}^{n}$ with $m \geq n$. The most important novelty is that the energy features a singular set $S_{u}$ of codimension greater than one, defined through the theory of distributional jacobians. After recalling the basic definitions and some well established results, we prove an approximation property for the energy $E(u, \Omega)$ via $\Gamma$-convergence, in the same spirit of the work by Ambrosio and Tortorelli [L. Ambrosio and V.M. Tortorelli, Commun. Pure Appl. Math. 43 (1990) 999-1036].
\end{abstract}

Résumé. Dans cet article on considère une nouvelle fonctionnelle du type de Mumford-Shah $E(u, \Omega)$ pour des applications $u: \mathbb{R}^{m} \rightarrow \mathbb{R}^{n}$ avec $m \geq n$. La nouveauté principale est que l'énergie présente un ensemble singulier $S_{u}$ de codimension supérieure à un, défini par la théorie des déterminant au sense de distributions. Après avoir rappelé les définitions de base et certains résultats classiques, nous prouvons une propriété d'approximation pour l'énergie $E(u, \Omega)$ par $\Gamma$-convergence, dans le même esprit de Ambrosio et Tortorelli [L. Ambrosio and V.M. Tortorelli, Commun. Pure Appl. Math. 43 (1990) 999-1036].

Mathematics Subject Classification. 49Q20, 49J45, 49Q15.

Received January 24, 2013. Revised April 5, 2013.

Published online January 27, 2014.

\section{INTRODUCTION}

The objects and the results of this paper belong to a larger research project on the fundamental properties of distributional jacobians. In this work we continue the study of a new functional in the calculus of variations of Mumford-Shah type $[6,19,38]$ started in [10], where the minimization involves an unknown function as well as a set:

$$
\mathcal{A}(u, K ; \Omega)=\int_{\Omega \backslash K} f(x, u, M \nabla u) \mathrm{d} x+\int_{\Omega \cap K} g \mathrm{~d} \mathscr{H}^{m-n} .
$$

Here $\Omega \subset \mathbb{R}^{m}$ is a bounded open set of class $C^{1}, u \in C^{1}\left(\Omega \backslash K, \mathbb{R}^{n}\right), M \nabla u$ is the vector of minors of $\nabla u$ of every rank, $\mathscr{H}^{m-n}$ is the $(m-n)$-dimensional Hausdorff measure and $K$ is a sufficiently regular closed set. The main novelty in this type of energies with respect to the classical Mumford-Shah energies $[8,18,38]$ is the presence of a "free discontinuity" set of codimension higher than one.

\footnotetext{
Keywords and phrases. Jacobian, $\Gamma$-convergence, higher codimension, Mumford-Shah, Ginzburg-Landau, phase transition.

1 Scuola Normale Superiore di Pisa, Piazza dei Cavalieri 7, 56126, Pisa, Italy. f.ghiraldin@sns.it
} 
In this paper we are concerned with the model case

$$
E(u, \Omega)=\int_{\Omega}|\nabla u|^{p}+\left|M_{n} \nabla u\right|^{\gamma} \mathrm{d} x+\sigma \mathscr{H}^{m-n}\left(\Omega \cap S_{u}\right)
$$

which we present already in the weak formulation: this energy features a new class of vector valued maps called $G S B_{n} V(\Omega)$, whose definition is related to the concept of distributional jacobian Ju. Here $u$ is a Sobolev map, $S_{u}$ is the singular set of its distributional jacobian $J u$, see Section 2 for the precise definitions. The simplified idea, in the special case $m=n$, is that $u$ is a vector-valued map regular outside a finite number of points where the map covers a set of positive measure, thus imposing a singularity to its jacobian. The functional penalizes maps with an excessively large area factor $M_{n} \nabla u=\operatorname{det} \nabla u$ as well as the creation of too large singular sets $S_{u}$. Note that the $p$ th power of the gradient helps smoothing possible wild oscillations of $u$, however if $p<n$ the map might still have a singular jacobian. Moreover like in [38] a lower semicontinuous fidelity term $\int_{\Omega}|u-g|^{r} \mathrm{~d} x$, forcing $u$ to be close to a given map $g$, can be added to (1.1).

The class $G S B_{n} V$ can be interpreted as a generalization of the well known function spaces $S B V$ and $B_{n} V$ (see $[8,31])$, where the differential $D u$ is replaced by $J u$ and where the jacobian is allowed to have infinite mass. Its definition takes deeply advantage of the slicing procedure available for flat currents, as it is well documented in $[9,11,20,22,24,42] . G S B_{n} V$ consists of Sobolev functions $u$ whose jacobian can be written as a sum of a finite mass flat current $R_{u}$ whose total variation is absolutely continuous with respect to Lebesgue measure, and a flat current $T_{u}$ of finite size. We devote part of Section 2 to describe this construction and compare it with the finite mass space $B_{n} V$.

Note that similarly to the codimension one case $[8,18]$ the finiteness of the energy does not imply any boundedness of the multiplicity density $\Theta^{m-n}(\|J u\|, \cdot)$ with respect to the Hausdorff measure $\mathscr{H}^{m-n}\left\llcorner S_{u}\right.$ : therefore $E(\cdot, \Omega)$ demands an adapted compactness and lower semicontinuity Theorem to show the existence of minimizers of suitable Dirichlet and Neumann problems. This result was obtained in [10], along with several examples and phenomenologies.

Recall the centrality of the distributional jacobian in the literature of Ginzburg-Landau problems, where the defects of constrained Sobolev maps are detected via the appearance of a singularity in $J u$, and where approximation results similar to ours have been obtained, see $[3,4,15,23,28,39]$. Another research field revolving around weak notions of area deformation is nonlinear elasticity, where the deformation $u$ of a material is driven by the energy minimization of a functional depending on the minors of $\nabla u$. The groundbreaking work [14] has been followed by a rich literature, where several theories treating possible formation of fractures and cavitations are described, see $[1,27,28,37,40]$.

In this paper we discuss a variational approximation of $E$ via $\Gamma$-convergence by (degenerate) elliptic functionals $E_{\varepsilon}$, in the spirit of $[12,13]$. These densities, being absolutely continuous, are easier to handle from the numerical viewpoint. Similarly to the scalar Mumford-Shah functional, we are able to approximate the defect measure, which is singular, via a family of bulk functionals (although not uniformly elliptic), a phenomenon already outlined in the pioneering papers by Modica and Mortola [33,34].

We want to approximate the maps $u \in G S B_{n} V$ with functions $u_{\varepsilon}$ possessing "better regularity", namely having absolutely continuous jacobian. Our choice of approximating functionals is

$$
E_{\varepsilon}(u, v, \Omega)=\int_{\Omega}|\nabla u|^{p}+\left(v+k_{\varepsilon}\right)\left|M_{n} \nabla u\right|^{\gamma} \mathrm{d} x+\int_{\Omega} \varepsilon^{q-n}|\nabla v|^{q}+\frac{W(1-v)}{\varepsilon^{n}} \mathrm{~d} x,
$$

and the limit takes place for $\varepsilon \rightarrow 0$. In (1.2) $v$ is a control function for the pointwise determinant $M_{n} \nabla u$, ranging in the interval $[0,1]$, and depends on the singular set $S_{u} ; k_{\varepsilon}$ is an infinitesimal number apt to guarantee coercivity of $E_{\varepsilon}$. The second integral, referred to as the Modica-Mortola term because of the similarity with the phase transition energies contained in [12], contains a nonnegative convex potential $W$ vanishing in 0 .

After a brief analysis on the existence of minimizers for $E_{\varepsilon}$ we proceed to show the main convergence result. The approximation of $E$ via $E_{\varepsilon}$ takes place in the sense of $\Gamma$-convergence, whose main properties are summarized 
at the beginning of Section 3. In particular the fundamental Theorem for such convergence yields:

$$
\left(u_{\varepsilon}, v_{\varepsilon}\right) \text { minimizes } E_{\varepsilon}, \quad\left(u_{\varepsilon}, v_{\varepsilon}\right) \rightarrow(u, v) \quad \Rightarrow \quad(u, v) \text { minimizes } E .
$$

As $\varepsilon$ goes to 0 , the potential term $W(1-v)$ forces $v_{\varepsilon}$ to converge to 1 in measure; on the contrary $v_{\varepsilon}$ becomes closer to 0 where the jacobian of the functions $u_{\varepsilon}$ tends to form a singularity, and compensates the loss of energy due to this damping with the Modica-Mortola term. Because of the scaling property of the Modica-Mortola part the transition from $v_{\varepsilon} \sim 0$ to $v_{\varepsilon} \sim 1$ happens in a set of width of order $\varepsilon$, and up to a rescaling $v_{\varepsilon}$ converges to a precise profile $w_{0}$ analysed in Section 4 . In particular this transition energy concentrates around the singular set $S_{u}$ proportionally to its $\mathscr{H}^{m-n}$-measure.

The proof of the approximation will be carried out in two steps: first we show

$$
\liminf _{\varepsilon \rightarrow 0} E_{\varepsilon}\left(u_{\varepsilon}, v_{\varepsilon}, \Omega\right) \geq E(u, \Omega)
$$

whenever $\left(u_{\varepsilon}, v_{\varepsilon}\right) \rightarrow(u, 1)$. This step is achieved first in codimension $m-n=0$, where $S_{u}$ is a discrete set, and then generalized to every codimension with the help of the slicing Theorem. The second part of the proof concerns the upper limit: here we construct $\left(u_{\varepsilon}\right)$ truncating the function $u$ around the singularity $S_{u}$ and we use the optimal profile $w_{0}$ to build functions $v_{\varepsilon}$ such that $\left(u_{\varepsilon}, v_{\varepsilon}\right) \rightarrow(u, 1)$ and

$$
\limsup _{\varepsilon \rightarrow 0} E_{\varepsilon}\left(u_{\varepsilon}, v_{\varepsilon}, \Omega\right) \leq E(u, \Omega) .
$$

In order to make this construction we will assume a mild regularity assumption on the singular set, namely

$$
\limsup _{r \downarrow 0} \frac{\mathscr{L}^{m}\left(\left\{x \in \Omega: \operatorname{dist}\left(x, S_{u}\right) \leq r\right\}\right)}{\mathscr{L}^{n}\left(B_{1}^{n}\right) r^{n}}=\mathscr{H}^{m-n}\left(S_{u}\right) .
$$

In order to conclude the proof of the $\Gamma$-convergence of $E_{\varepsilon}$ to $E$ we would need to know the density in energy of the set of $G S B_{n} V$ maps satisfying (1.3). In the codimension 1 case this property was deduced by the regularity of minimizers of the Mumford-Shah energy, for which a lower bound on the $(m-1)$-dimensional density of the singular set is available. The analogous density property as well as a regularity result for minimizers of $E$ will be subject to further investigation.

In Section 7 we prove an analog approximation result where we impose a fixed boundary condition to both $u$ and the approximating sequence $\left(u_{\varepsilon}\right)$. In the case $S_{u} \cap \partial \Omega \neq \emptyset$ then the transition made by $v$ takes place partially outside the domain $\Omega$, which translates in a loss of mass in the limit energy.

Finally in the last section we discuss a possible generalization to general Lagrangians, featuring a polyconvex integrand for the bulk part and where the size term is weighted by a continuous density. Growth and convexity assumptions will be crucial to extend the results of the previous sections to this broader class of energies.

\section{Distributional JACOBians}

We begin by fixing some basic notions and recalling some properties of distributional jacobians: we will assume, if not otherwise specified, that $\Omega$ is a bounded open subset of $\mathbb{R}^{m}$ with boundary of class $C^{1}$, that $m \geq n$ are positive integers and that $p$ and $s$ are positive exponents satisfying

$$
\frac{1}{s}+\frac{n-1}{p} \leq 1, \quad s<\infty:
$$

observe that this limitation allows $p$ to be smaller than the critical exponent $n$.

As customary the symbol $\Lambda_{k} \mathbb{R}^{m}$ will denote the space of $k$-vectors of $\mathbb{R}^{m}$. We will let

$$
\mathbf{O}_{k}=\left\{L: \mathbb{R}^{m} \rightarrow \mathbb{R}^{m}: L=L^{t}, L^{2}=L, \operatorname{rk}(L)=k\right\}
$$


be the space of orthogonal projections of rank $k$, for $1 \leq k \leq m$. Given a linear map $L: \mathbb{R}^{m} \rightarrow \mathbb{R}^{n}$ we adopt for the collection $M_{k} L$ of determinants of $k \times k$ minors of $L$ the following sign convention:

$$
M_{k} L:=\left(e_{1} \wedge \ldots \wedge e_{m} L L^{i_{1}} \wedge \ldots \wedge L^{i_{k}}\right)_{\left\{i_{1}<\ldots<i_{k}\right\} \subset\{1, \ldots, n\}} .
$$

In this way we group the minors with the same rows in a single element of $\Lambda_{m-k} \mathbb{R}^{m}$. We let $M L=$ $\left(M_{1} L, \ldots, M_{n} L\right)$ be the vector minors of every rank; $\kappa:=\sum_{k=1}^{n}\left(\begin{array}{c}m \\ k\end{array}\right)\left(\begin{array}{c}n \\ k\end{array}\right)$ will be its dimension. Given $w \in \mathbb{R}^{\kappa}$ we let $w_{k}$ be the variables relative to $k \times k$ minors. For our purposes we will need to measure the length of $\nu \in \Lambda_{k} \mathbb{R}^{m}$ so that

$$
|\nu|=\sup _{\pi \in \mathbf{O}_{k}} \mid \nu\left\llcorner\mathrm{d} \pi\left|=\sup _{\pi \in \mathbf{O}_{k}}\right|\langle\mathrm{d} \pi, \nu\rangle \mid:\right.
$$

it can be proved that the Euclidean norm satisfies this property, see $[10,24]$.

Weak convergence in the $L^{p}$ spaces will be customarily denoted with the symbol $\rightarrow$ : in particular in the non-reflexive case $p=1$ this is the convergence against fixed $L^{\infty}$ functions. Sobolev maps $u: \Omega \subset \mathbb{R}^{m} \rightarrow \mathbb{R}^{n}$ are known to possess an approximate differential $\nabla u(x) \in \mathbb{R}^{n \times m}$ at almost every point $x \in \Omega$, see [43], Theorem 3.4.2.

We will denote by $\mathbf{F}_{k}(\Omega)$ and $\mathbf{M}_{k}(\Omega)$ the spaces respectively of flat and finite mass $k$-dimensional currents in an open subset $\Omega \subset \mathbb{R}^{m}$ (see $[9,24,26]$ ). The action of a current $T$ on a differential form $\psi$ will be denoted by $\langle T, \psi\rangle$, and weak* convergence (that is: pointwise convergence of the functionals $\left\langle T_{h}, \psi\right\rangle \rightarrow\langle T, \psi\rangle$ for every fixed compactly supported smooth differential form $\psi$ ) will be denoted by $\stackrel{*}{\rightarrow}$. The same notation is adopted for weak* convergence of measures. The top dimensional $m$-current representing the Lebesgue integration with the standard orientation on $\mathbb{R}^{m}$ will be denoted by $\mathbf{E}^{m}$ :

$$
\mathbf{E}^{m}\left(\varphi \mathrm{d} x^{1} \wedge \ldots \wedge \mathrm{d} x^{m}\right)=\int_{\mathbb{R}^{m}} \varphi(x) \mathrm{d} \mathscr{L}^{m}(x) .
$$

To our knowledge the notions of distributional jacobian and $B_{n} V$ function were defined first in [31]: the basic necessary assumption on $u$ to give this definition is membership to $\dot{W}^{1, p} \cap L^{s}=\left\{u \in L^{s}, \nabla u \in L^{p}\right\}$.

Definition 2.1 (distributional jacobian and $B_{n} V$ functions). Let $u \in \dot{W}^{1, p} \cap L^{s}\left(\Omega, \mathbb{R}^{n}\right)$. We denote by $j(u)$ the $(m-n+1)$-current

$$
\langle j(u), \omega\rangle:=(-1)^{n} \int_{\Omega} u^{1} \mathrm{~d} u^{2} \wedge \ldots \wedge \mathrm{d} u^{n} \wedge \omega,
$$

where $\omega$ is a smooth $(m-n+1)$-form with compact support in $\Omega$; we define the distributional jacobian of $u$ as the $(m-n)$-dimensional flat current

$$
J u:=\partial j(u) \in \mathbf{F}_{m-n}(\Omega) .
$$

We say that a map $u \in \dot{W}^{1, p} \cap L^{s}$ belongs to $B_{n} V$ if its distributional jacobian $J u$ has finite mass (and hence it can be represented by a Radon measure).

Few observation are in order: first of all the integrability assumption $u \in \dot{W}^{1, p} \cap L^{s}$ ensures that (2.3) is welldefined; observe that for $p \geq \frac{m n}{m+1} J u$ is defined for $u \in W^{1, p}$, since in this case $W^{1, p} \subset L^{s}$ for some sufficiently large exponent $s$ satisfying (2.1), by Sobolev embedding. Notice also that this constraint allows the case $p<n$. Since $j(u)$ is explicitly represented as the integration against a $\Lambda_{m-n}$-valued $L^{1}$ function, $J u$ belongs to the space of flat currents $\mathbf{F}_{m-n}(\Omega)$ (see [24], 4.1.18 and [10] for a proof of this fact). Regarding the convergence properties of jacobians, we consider the following flat norm on $k$-currents

$$
\mathbf{F}(T):=\sup \left\{\langle T, \psi\rangle: \psi \in C_{c}^{\infty}\left(\Omega, \Lambda^{k} \mathbb{R}^{m}\right), \max \left\{\|\psi\|_{\infty},\|\mathrm{d} \psi\|_{\infty}\right\} \leq 1\right\}:
$$

given $u_{h}, u \in \dot{W}^{1, p} \cap L^{s}\left(\Omega, \mathbb{R}^{n}\right)$ we have

$$
u_{h} \rightarrow u \text { in } L^{s}\left(\Omega, \mathbb{R}^{n}\right), \quad \nabla u_{h} \rightarrow \nabla u \text { in } L^{p}\left(\Omega, \mathbb{R}^{n \times m}\right) \quad \Rightarrow \quad \mathbf{F}\left(J u_{h}-J u\right) \rightarrow 0,
$$


hence weakly* in the sense of currents, compare [10]. If moreover $\left(u_{h}\right) \subset B_{n} V$ and $\mathbf{M}\left(J u_{h}\right)=\left\|J u_{h}\right\|(\Omega) \leq$ $C<\infty$ then $u \in B_{n} V$ and the convergence takes place in the sense of measures. In particular if $p \geq n$, by convolution every function $u \in \dot{W}^{1, p} \cap L^{s}$ has a sequence $\left(u_{h}\right) \subset C^{\infty}$ approximating $u$ strongly in the Sobolev space $W_{\text {loc }}^{1, n}$ : since $\omega$ has compact support passing to the limit in the integration by parts formula

$$
\left\langle J u_{h}, \psi\right\rangle=(-1)^{n} \int_{\Omega} u_{h}^{1} \mathrm{~d} u_{h}^{2} \wedge \ldots \wedge \mathrm{d} u_{h}^{n} \wedge \mathrm{d} \psi=\int_{\Omega} \mathrm{d} u_{h}^{1} \wedge \mathrm{d} u_{h}^{2} \wedge \ldots \wedge \mathrm{d} u_{h}^{n} \wedge \psi
$$

we obtain that $J u=\mathbf{E}^{m}\left\llcorner(-1)^{n(m-n)} \mathrm{d} u^{1} \wedge \ldots \wedge \mathrm{d} u^{n}\right.$. In particular if the gradient $\nabla u$ has a sufficiently high summability, then $J u$ is an absolutely continuous measure. On the other hand when $p<n$ there are several examples of functions whose jacobian is not in $L^{1}$ : for instance the "monopole" function $u(x):=\frac{x}{|x|}$ satisfies $J u=\mathscr{L}^{n}\left(B_{1}\right) \llbracket 0 \rrbracket$, where $0 \rrbracket$ is the Dirac mass in the origin. More complicated examples, including maps such that $J u$ has infinite mass or such that $J u$ is not even a Radon measure, are presented in $[3,10,31,37]$. We finally remark that in our paper membership to $B_{n} V$, or to any other space whose definition involves $J u$, implicitly assumes $u \in \dot{W}^{1, p} \cap L^{s}$, for $p, s$ as in (2.1).

Distributional jacobians of $B_{n} V$ functions, being $\Lambda_{m-n}$-valued measures, satisfy a decomposition in three mutually singular parts (see $[8,20,31]$ ):

$$
J u=\nu \cdot \mathscr{L}^{m}+J^{c} u+\theta \cdot \mathscr{H}^{m-n} \mathrm{~L} S_{u}
$$

where

- $\nu=\frac{d J u}{\mathrm{~d} \mathscr{L}^{m}} \in L^{1}\left(\Omega, \Lambda_{m-n}\left(\mathbb{R}^{m}\right)\right)$ is the Radon Nikodym derivative of $J u$ with respect to $\mathscr{L}^{m}$;

- $\theta \in L^{1}\left(\Omega, \Lambda_{m-n}\left(\mathbb{R}^{m}\right), \mathscr{H}^{m-n}\right)$ is a measurable function and $S_{u}$ is a $\mathscr{H}^{m-n} \sigma$-finite subset of $\Omega$;

- $\left\|J^{c} u\right\|(F)=0$ whenever $\mathscr{H}^{m-n}(F)<\infty$.

It can be proved that

$$
\nu(x)=M_{n} \nabla u(x)=e_{1} \wedge \ldots \wedge e_{m} L \mathrm{~d} u^{1} \wedge \ldots \wedge \mathrm{d} u^{n} \in \Lambda_{m-n}\left(\mathbb{R}^{m}\right)
$$

at $\mathscr{L}^{m}$-almost every point $x \in \Omega$ (see $[21,36]$ ). The set $S_{u}$ is unique up to $\mathscr{H}^{m-n}$-negligible sets, by intersecting it with $\{|\theta|>0\}$; moreover $S_{u}$ is $\mathscr{H}^{m-n}$-countably rectifiable (see [20]). In analogy with the codimension one case we denote $S B_{n} V(\Omega)$ the subset of $B_{n} V(\Omega)$ of functions such that $J^{c} u=0$. This space enjoys a closure property proved in [20]:

Theorem 2.2 (closure theorem for $\left.S B_{n} V\right)$. Let us consider $u, u_{k} \in B_{n} V\left(\Omega, \mathbb{R}^{n}\right)$ with $\Omega \subset \mathbb{R}^{m}$ and suppose that

(a) $u_{h} \rightarrow u$ strongly in $L^{s}\left(\Omega, \mathbb{R}^{n}\right)$ and $\nabla u_{h} \rightarrow \nabla u$ weakly in $L^{p}\left(\Omega, \mathbb{R}^{n \times m}\right)$,

(b) if we write

$$
J u_{h}=\nu_{h} \cdot \mathscr{L}^{m}+\theta_{h} \cdot \mathscr{H}^{m-n}\left\llcorner S_{u_{h}}\right.
$$

then $\left|\nu_{h}\right|$ are equiintegrable in $\Omega$ and $\mathscr{H}^{m-n}\left(S_{u_{h}}\right) \leq C<\infty$.

Then $u \in S B_{n} V\left(\Omega, \mathbb{R}^{n}\right)$ and

$$
\nu_{h} \rightarrow \nu \text { weakly in } L^{1}\left(\Omega, \Lambda_{m-n}\left(\mathbb{R}^{m}\right)\right), \quad \mathscr{H}^{m-n}\left(S_{u}\right) \leq \liminf _{h} \mathscr{H}^{m-n}\left(S_{u_{h}}\right) .
$$

As explained in [24], 4.2 and [9], every flat current $T \in \mathbf{F}_{k}(\Omega)$ can be sliced via a Lipschitz map $\pi \in$ $\operatorname{Lip}\left(\Omega, \mathbb{R}^{\ell}\right), \ell \leq k$ : the result is a collection of currents

$$
\langle T, \pi, x\rangle \in \mathbf{F}_{k-\ell}(\Omega) \quad \text { defined for } \mathscr{L}^{\ell} \text {-a.e. } x \in \mathbb{R}^{\ell}
$$


satisfying several properties. Amongst them we recall

$$
\begin{aligned}
& T\left\llcorner d \pi=\int_{\mathbb{R}^{\ell}}\langle T, \pi, x\rangle \mathrm{d} \mathscr{L}^{\ell}(x),\right. \\
& \langle T, \pi, x\rangle \text { is concentrated on } \pi^{-1}(x) \text { for } \mathscr{L}^{\ell} \text {-a.e. } x \in \mathbb{R}^{\ell}, \\
& \int_{\mathbb{R}^{\ell}} \mathbf{F}(\langle T, \pi, x\rangle) \mathrm{d} \mathscr{L}^{\ell}(x) \leq \operatorname{Lip}(\pi)^{\ell} \mathbf{F}(T),
\end{aligned}
$$

and we refer to [24], 4.2.1 and to [11] for a general account in the Euclidean and general metric setting. We aim to apply this operation to $J u \in \mathbf{F}_{m-n}(\Omega)$ in the special case $\ell=m-n$, thus reducing it to 0-dimensional slices; moreover we want to relate those slices to the jacobian of the restriction $J\left(\left.u\right|_{\pi^{-1}(x)}\right)$. Let therefore $\pi \in \mathbf{O}_{m-n}$ : for each $x \in \pi\left(\mathbb{R}^{m}\right)$ we let $i^{x}: \mathbb{R}^{n} \rightarrow \mathbb{R}^{m}$ be the orthogonal injection of $\mathbb{R}^{n}$ onto $\pi^{-1}(x)$. In [20], the author proved the following slicing Theorem for jacobians:

Theorem 2.3 (Slicing). Let $u \in \dot{W}^{1, p} \cap L^{s}\left(\Omega, \mathbb{R}^{n}\right)$ and let $\pi \in \mathbf{O}_{m-n}$. Then for $\mathscr{L}^{m-n}$-almost every $x \in \mathbb{R}^{m-n}$

$$
\langle J u, \pi, x\rangle=(-1)^{(m-n) n} i_{\#}^{x}\left(J u^{x}\right),
$$

where $u^{x}=u \circ i^{x}$. Moreover $u \in B_{n} V\left(\Omega, \mathbb{R}^{n}\right)$ if and only if for every $\pi \in \mathbf{O}_{m-n}$ the following two conditions hold:

$$
\begin{aligned}
& u^{x} \in B_{n} V\left(\Omega^{x}, \mathbb{R}^{n}\right) \quad \text { for } \quad \mathscr{L}^{m-n} \text {-almost every } x \in \mathbb{R}^{m-n}, \\
& \int_{\pi(\Omega)}\|\langle J u, \pi, x\rangle\|\left(\Omega^{x}\right) \mathrm{d} \mathscr{L}^{m-n}(x)<\infty,
\end{aligned}
$$

where $\Omega^{x}=\Omega \cap \pi^{-1}(x)$. If $u \in B_{n} V\left(\Omega, \mathbb{R}^{n}\right)$ the slicing property (2.5) holds separately for the absolutely continuous part, the Cantor part and the jump part of Ju, namely:

- $\left\langle J^{a} u, \pi, x\right\rangle=(-1)^{(m-n) n} i_{\#}^{x}\left(J^{a} u^{x}\right)$,

- $\left\langle J^{c} u, \pi, x\right\rangle=(-1)^{(m-n) n} i_{\#}^{x}\left(J^{c} u^{x}\right)$,

- $\left\langle J^{j} u, \pi, x\right\rangle=(-1)^{(m-n) n} i_{\#}^{x}\left(J^{j} u^{x}\right)$.

Since we will work with functions whose jacobian does not have a Cantor part, it is useful to notice that in order to check that some function $u$ belongs to $S B_{n} V$ it is sufficient to check that, along with the integrability assumption (ii), for almost every slice $J u^{x}$ has no Cantor part. Moreover in the general theory of current in metric spaces the bound (ii) would be required uniform in $\pi$, see [7,10]; in the Euclidean space it is of course enough to check such property for $\left(\begin{array}{c}m \\ n\end{array}\right)$ linearly independent projections.

\subsection{A new class of maps related to size}

In order to study a minimization problem it is necessary to consider, along with the topology, the natural domain of the functional, and to understand the potential limit points of energy-bounded sequences. As anticipated in the introduction our functional $E$ penalizes the size of the singular set of $J u$, regardless of the multiplicity function $\theta$. This lack of control on the mass of $J u$, which already appears in Theorem 2.2 when we require $u \in B_{n} V$, forces us to extend the notion of admissible maps beyond $B_{n} V$, through the concept of size. In general it is possible to define a measure-theoretic quantity $\mathbf{S}(T)$, called size of $T$, for flat currents $T \in \mathbf{F}_{k}(\Omega)$ with possibly infinite mass. This quantity was introduced in [9], borrowing some ideas already used by Hardt and Rivière in [30] and Almgren [5] and agrees with the classical notion of size for finite mass currents, namely

$$
\mathbf{S}(T)=\mathscr{H}^{m-n}\left(\left\{\Theta^{m-n}(\|T\|, \cdot)>0\right\}\right), \quad T \in \mathbf{M}_{k}(\Omega) \cap \mathbf{F}_{k}(\Omega)
$$

as in [25]. The main idea behind this to detect the support of the 0-dimensional slices of $T$ and then to optimize the choice of projection $\pi$. 
Definition 2.4 (size of a flat current). We say that $T \in \mathbf{F}_{k}(E)$ has finite size if there exists a positive Borel measure $\mu$ such that

$$
\begin{aligned}
& \begin{array}{ll}
\mathscr{H}^{0} L \operatorname{spt}(T) \leq \mu & \text { for } k=0,
\end{array} \\
& \mu_{T, \pi}:=\int_{\mathbb{R}^{k}} \mathscr{H}^{0} \mathrm{~L} \operatorname{spt}\langle T, \pi, x\rangle \mathrm{d} \mathscr{L}^{k}(x) \leq \mu \quad \forall \pi \in \mathbf{O}_{k} \quad \text { for } k \geq 1 .
\end{aligned}
$$

The choice of $\mu$ can be optimized by choosing the least upper bound of the family $\left\{\mu_{T, \pi}\right\}$ in the lattice of nonnegative measures:

$$
\mu_{T}:=\bigvee_{\pi \in \mathbf{O}_{k}} \mu_{T, \pi}=\bigvee_{\pi \in \mathbf{O}_{k}} \int_{\mathbb{R}^{k}} \mathscr{H}^{0} \operatorname{Lspt}\langle T, \pi, x\rangle \mathrm{d} \mathscr{L}^{k}(x)
$$

We set $\mathbf{S}(T):=\mu_{T}(\Omega)$.

It can be proved (see [9]) that every flat $k$-current with finite size has a unique (up to null sets) countably $\mathscr{H}^{k}$-rectifiable set called $\operatorname{set}(T)$ where $\mu_{T}$ is concentrated, which satisfies $\mathscr{H}^{k}(\operatorname{set}(T))=\mathbf{S}(T)$. The reader can find an example of flat current having finite size but infinite mass in $[10,35]$.

The natural space for our problem is the set of Sobolev functions $u$ with the integrability expressed in (2.1), whose jacobian can be split in the sum of two parts:

- one is an $m$-dimensional current $R$ of finite mass, such that the measure $\|R\|$ is absolutely continuous with respect to $\mathscr{L}^{m}$;

- the other one is an $(m-n)$-dimensional flat chain $T$ of finite size.

Definition 2.5 (functions of special jacobian). The space of function of special jacobian is

$$
G S B_{n} V(\Omega)=\left\{u \in \dot{W}^{1, p} \cap L^{s}\left(\Omega, \mathbb{R}^{n}\right): J u=R+T, \mathbf{M}(R)+\mathbf{S}(T)<\infty,\|R\| \ll \mathscr{L}^{m}\right\} .
$$

This space is clearly meant to mimic the aforementioned $S B_{n} V$ class. Thanks to the relation between the slices of $J u$ and the jacobian of the restrictions expressed by (2.5), if $u \in G S B_{n} V(\Omega)$ and $\pi \in \mathbf{O}_{m-n}$ we can observe that for almost every $x$ the slice $\left\langle R_{u}, \pi, x\right\rangle$ has finite mass and is absolutely continuous with respect to $\mathscr{H}^{n}\left\llcorner\pi^{-1}(x)\right.$, while by Definition $2.4 \mathbf{S}\left(\left\langle T_{u}, \pi, x\right\rangle\right)<\infty$. Therefore $u^{x} \in G S B_{n} V\left(\Omega^{x}\right)$ for $\mathscr{L}^{m-n}$-almost every $x \in \mathbb{R}^{m-n}$. In the following propositions we describe some useful properties of the class $G S B_{n} V(\Omega)$.

Proposition 2.6 ([10], Lem. 3.0.5). If $m=n$ then $G S B_{n} V(\Omega)=S B_{n} V(\Omega)$.

The last Proposition shows that the difference between the spaces $G S B_{n} V(\Omega)$ and $S B_{n} V(\Omega)$ relies on the failure of the integrability condition (ii) in Theorem 2.3. Moreover, since the Radon-Nikodym decomposition of a measure into the sum of an absolutely continuous and a singular part is unique, by slicing also $R$ and $T$ are uniquely determined in the decomposition. Therefore we can write $J u=R_{u}+T_{u}$, so that $\operatorname{set}\left(T_{u}\right)$ is a well defined countably $\mathscr{H}^{m-n}$-rectifiable set. In agreement with the scalar case $n=1$ we let

$$
S_{u}:=\operatorname{set}\left(T_{u}\right)
$$

be the singular set of the map $u$. Moreover the pointwise characterization of $R_{u}$ also holds for $G S B_{n} V$ maps.

Proposition 2.7 (Det $=\operatorname{det}$ in the $G S B_{n} V$ class, [10], Prop. 3.2.1). Let $u \in G S B_{n} V(\Omega)$ and write Ju= $R_{u}+T_{u}$ as in Definition 2.5. Then $\mathscr{L}^{m}$-almost everywhere

$$
\frac{\mathrm{d} R_{u}}{\mathrm{~d} \mathscr{L}^{m}}=M_{n} \nabla u
$$


The fundamental Theorem on the space $G S B_{n} V$ is the following compactness result:

Theorem 2.8 (compactness for the class $G S B_{n} V,[10]$, Thm. 4.0.3). Let $\Psi:[0, \infty) \rightarrow[0, \infty)$ be a convex increasing function satisfying $\lim _{t \rightarrow \infty} \Psi(t) / t=\infty$. Let $\left(u_{h}\right) \subset G S B_{n} V(\Omega)$ satisfy $u_{h} \rightarrow u$ in $L^{s}\left(\Omega, \mathbb{R}^{n}\right)$ and $\nabla u_{h} \rightarrow \nabla u$ weakly in $L^{p}\left(\Omega, \mathbb{R}^{n \times m}\right)$. Assume that the jacobians $J u_{h}=R_{u_{h}}+T_{u_{h}}$ satisfy

$$
\sup _{h} \int_{\Omega} \Psi\left(\left|\frac{\mathrm{d} R_{u_{h}}}{\mathrm{~d} \mathscr{L}^{m}}\right|\right) \mathrm{d} \mathscr{L}^{m}+\mathbf{S}\left(T_{u_{h}}\right)<\infty .
$$

Then $u \in G S B_{n} V(\Omega)$ and, writing $J u=R_{u}+T_{u}$,

$$
\begin{aligned}
& \frac{\mathrm{d} R_{u_{h}}}{\mathrm{~d} \mathscr{L}^{m}} \rightarrow \frac{\mathrm{d} R_{u}}{\mathrm{~d} \mathscr{L}^{m}} \quad \text { weakly in } L^{1}\left(\Omega, \Lambda_{m-n} \mathbb{R}^{m}\right), \\
& \mathbf{S}\left(T_{u}\right) \leq \liminf _{h} \mathbf{S}\left(T_{u_{h}}\right) .
\end{aligned}
$$

In the sequel it will be handier to have a name for the space of function of bounded $n$-variation with absolutely continuous jacobian:

Definition 2.9 (regular maps). We let

$$
R_{n}(\Omega):=\left\{u \in B_{n} V(\Omega):\|J u\| \ll \mathscr{L}^{m}\right\}
$$

be the space of regular maps.

We have now all the elements to define our Mumford-Shah energy of codimension higher than one.

Definition 2.10. Let $\gamma>1$ and $\sigma>0$. For every $u \in G S B_{n} V(\Omega)$ we set

$$
E(u, \Omega)=\int_{\Omega}|\nabla u|^{p}+\left|M_{n} \nabla u\right|^{\gamma} \mathrm{d} x+\sigma \mathscr{H}^{m-n}\left(\Omega \cap S_{u}\right) .
$$

It has been proved in [10] the following existence theorem, even for a broader class of Lagrangians, and for several notions of boundary conditions. Here we report the version most suitable to the scope of this paper.

Theorem 2.11 (existence of minimizers for the Dirichlet and Neumann problems). Let $\Omega$ be a regular open and bounded subset of $\mathbb{R}^{m}$ and let $U$ be an open neighborhood of $\bar{\Omega}$. Let $\phi \in G S B_{n} V(U)$ be a given function and suppose $p^{*}=\frac{m p}{m-p}>s$. Then the minimum problem

$$
\inf \left\{E(u, \bar{\Omega}): u \in G S B_{n} V(U), u=\phi \text { in } U \backslash \Omega\right\}
$$

has a solution. Similarly for the Neumann problem if $r>s$ and $g \in L^{r}\left(\Omega, \mathbb{R}^{n}\right)$ is given, then

$$
\inf \left\{E(u, \Omega)+\int_{\Omega}|u-g|^{r} \mathrm{~d} x: u \in G S B_{n} V(\Omega)\right\}
$$

has a solution.

\subsection{Minkowski content}

As Theorem 3.7 below involves the concept of Minkowski content, we here briefly review its definition and main properties. 
Definition 2.12. Let $S \subset \mathbb{R}^{m}$ and let $k \in[0, m]$ be and integer. The lower and upper Minkowski contents of $S$ in $\Omega$ are defined respectively as

$$
\begin{aligned}
& \mathcal{M}_{* \Omega}^{k}(S)=\liminf _{r \downarrow 0} \frac{\mathscr{L}^{m}(\{x \in \Omega: \operatorname{dist}(x, S) \leq r\})}{\mathscr{L}^{m-k}\left(B_{1}\right) r^{m-k}}, \\
& \mathcal{M}_{\Omega}^{* k}(S)=\limsup _{r \downarrow 0} \frac{\mathscr{L}^{m}(\{x \in \Omega: \operatorname{dist}(x, S) \leq r\})}{\mathscr{L}^{m-k}\left(B_{1}\right) r^{m-k}},
\end{aligned}
$$

where $\mathscr{L}^{m-k}\left(B_{1}\right)$ is the measure of the unit ball in $\mathbb{R}^{m-k}$. We omit the subscript when $\Omega=\mathbb{R}^{m}$. If $\mathcal{M}_{*}^{k}(S)=$ $\mathcal{M}^{* k}(S)$ we define the Minkowski content of $S$ as this common value.

We must observe that neither $\mathcal{M}_{*}^{k}$ nor $\mathcal{M}^{* k}$ is a measure, and that they both give the same value to a set and its closure. It is natural to compare the upper and lower Minkowski contents with the $k$-dimensional Hausdorff measure: it can be proved (see [24], 3.2.37-39, [8], 2.101) that for every countably $\mathscr{H}^{k}$-rectifiable and closed set $S$

$$
\mathcal{M}_{*}^{k}(S) \geq \mathscr{H}^{k}(S)
$$

By inner regularity of the Hausdorff measure the last inequality holds also relative to $\Omega$. Various assumptions on $S$ besides rectifiability are possible in order to have that $\mathcal{M}^{k}(S)=\mathscr{H}^{k}(S)$. One of the most general is the following:

Proposition 2.13 ([8], Prop. 2.104). Let $S$ be a countably $\mathscr{H}^{k}$-rectifiable set such that

$$
\nu\left(B_{\rho}(x)\right) \geq c \rho^{k} \quad \forall x \in S \quad \forall \rho \in\left(0, \rho_{0}\right)
$$

for a suitable Radon measure $\nu \ll \mathscr{H}^{k}$ and $c, \rho_{0}>0$. Then

$$
\mathcal{M}^{k}(S)=\mathscr{H}^{k}(S)
$$

Note that the equality implies that $\mathscr{H}^{k}(S)=\mathscr{H}^{k}(\bar{S})$. To ease the notation we will denote $S_{r}=\{x \in \Omega: 0<$ $\operatorname{dist}(x, S) \leq r\}$ and $V(r)=\mathscr{L}^{m}\left(S_{r}\right)$. Let $S \subset \mathbb{R}^{m}$ be a closed set, and consider the distance function from it. Then (see [24], 3.2.34)

$$
|\nabla \operatorname{dist}(\cdot, S)|=1 \quad \mathscr{L}^{m} \text {-a.e. in }\{\operatorname{dist}(\cdot, S)>0\} .
$$

Moreover the following property holds:

Lemma 2.14. The function $V(t)=\mathscr{L}^{m}(\{0<\operatorname{dist}(\cdot, S) \leq t\})$ is absolutely continuous and

$$
V^{\prime}(t)=\mathscr{H}^{m-1}(\{x \in \Omega: \operatorname{dist}(x, S)=t\})
$$

for $\mathscr{L}^{1}$-almost every $t>0$.

Proof. Recall the Coarea formula [24], 3.2.11-12: if $f: \Omega \rightarrow \mathbb{R}$ is a Lipschitz function and $g: \Omega \rightarrow \mathbb{R}$ is a non-negative Borel function, then

$$
\int_{\Omega} g(x)|\nabla f(x)| \mathrm{d} x=\int_{0}^{+\infty} \int_{\{f=t\}} g \mathrm{~d} \mathscr{H}^{m-1} \mathrm{~d} t .
$$

In particular taking $f(x)=\operatorname{dist}(x, S)$ and $g$ the characteristic function of the set $\{\operatorname{dist}(\cdot, S) \leq t\}$ we obtain that for every $t>0$

$$
V(t)=\int_{0}^{t} \mathscr{H}^{m-1}(\Omega \cap\{\operatorname{dist}(\cdot, S)=s\}) \mathrm{d} s .
$$

Therefore $V(t)$ is an absolutely continuous function with

$$
V^{\prime}(t)=\mathscr{H}^{m-1}(\{x \in \Omega: \operatorname{dist}(x, S)=t\})
$$

$\mathscr{L}^{1}$-almost everywhere. 


\section{VARIATIONAL APPROXIMATION}

In this section we state our main approximation theorem. We start by recalling the fundamental features of the variational convergence we will use, the $\Gamma$-convergence, and we refer to $[16,17]$ for a thorough presentation. Let $X$ be a separable metric space and let a sequence of functions $F_{h}: X \rightarrow[0, \infty]$ be given. We define the upper and the lower $\Gamma$-limits as follows:

$$
\begin{gathered}
\underline{F}(x)=\left(\Gamma-\liminf _{h \rightarrow \infty} F_{h}\right)(x)=\inf \left\{\liminf _{h \rightarrow \infty} F_{h}\left(x_{h}\right): x_{h} \rightarrow x\right\}, \\
\bar{F}(x)=\left(\Gamma-\limsup _{h \rightarrow \infty} F_{h}\right)(x)=\inf \left\{\limsup _{h \rightarrow \infty} F_{h}\left(x_{h}\right): x_{h} \rightarrow x\right\} .
\end{gathered}
$$

Both $\underline{F}$ and $\bar{F}$ are lower semicontinuous by construction, and we say that $F_{h} \Gamma$-converges to $F$ if $\underline{F}=\bar{F}$. The statement $\Gamma-\lim _{h} F_{h}=F$ is equivalent to the fulfillment of the following two conditions: for every $x \in X$

$$
\begin{gathered}
\forall x_{h} \rightarrow x \text { we have } \liminf _{h} F_{h}\left(x_{h}\right) \geq F(x), \\
\exists x_{h} \rightarrow x \text { such that } \limsup _{h} F_{h}\left(x_{h}\right) \leq F(x) .
\end{gathered}
$$

The following Theorem describes the fundamental properties of this type of convergence, in particular the behaviour of sequences of minima:

Theorem 3.1. Assume $F_{h} \Gamma$-converges to $F$.

(a) Let $t_{h} \downarrow 0$. Then any cluster point of the sequence of sets

$$
\left\{x \in X: F_{h}(x) \leq \inf _{X} F_{h}+t_{h}\right\}
$$

minimizes $F$.

(b) Assume also that $F_{h}$ are lower semicontinuous, and that for every $t \geq 0$ there exists a compact set $K_{t} \subset X$ such that

$$
\left\{F_{h} \leq t\right\} \subset K_{t} .
$$

Then every function $F_{h}$ has a minimizer, and any sequence of minimizers admits a subsequence converging to some minimizer of $F$.

(c) Given a continuous function $G: X \rightarrow[0, \infty]$ we have

$$
\begin{aligned}
\Gamma-\liminf _{h}\left(F_{h}+G\right) & =\left(\Gamma-\liminf _{h} F_{h}\right)+G, \\
\Gamma-\limsup _{h}\left(F_{h}+G\right) & =\left(\Gamma-\limsup _{h} F_{h}\right)+G .
\end{aligned}
$$

The following remark recalls a useful tool in proving $\Gamma$-convergence results.

Remark 3.2. Let $X^{\prime} \subset X$ and $F, F_{h}: X \rightarrow \mathbb{R}$ as above: we say that $X^{\prime}$ is dense in energy in $X$ if for every $x \in X$ there exists a sequence $\left(x_{h}^{\prime}\right) \subset X^{\prime}$ such that $x_{h}^{\prime} \rightarrow x$ and $F\left(x_{h}^{\prime}\right) \rightarrow F(x)$. A simple diagonal argument shows that in order to prove $\Gamma-\lim F_{h}=F$, whilst already knowing the $\Gamma-\lim$ inf inequality $F \leq \underline{F}$ (namely the validity of (3.3)), it is enough to prove that for every $\delta>0$ and $x \in X^{\prime}$ there exists $x_{h} \rightarrow x$ such that $\lim \sup _{h} F_{h}\left(x_{h}\right) \leq F(x)+\delta$.

\subsection{Main theorem}

We introduce now the function spaces involved in our approximation Theorem. Given an open set $U \subset \mathbb{R}^{n}$ we let $B(U)$ be the space of Borel functions ranging in $[0,1]$ :

$$
B(U)=\{v: U \rightarrow[0,1]: v \text { is a Borel function }\},
$$


endowed with a distance that induces the convergence in measure, namely:

$$
d\left(v, v^{\prime}\right)=\int_{\Omega} \frac{\left|v-v^{\prime}\right|}{1+\left|v-v^{\prime}\right|} \mathrm{d} x .
$$

We want to approach the energy $E(u, \Omega)$ by a sequence $E_{h}\left(u_{h}, v_{h}, \Omega\right)$ where the functions $u_{h}$ belong to $R_{n}(\Omega)$, namely $J u_{h}=R_{u_{h}}=M_{n} \nabla u_{h} \mathscr{L}^{m}$. Our function spaces will be the following:

Definition 3.3. We define the space $X(\Omega):=L^{s}\left(\Omega, \mathbb{R}^{n}\right) \times B(\Omega)$ with the following convergence notion:

$$
\left(u_{h}, v_{h}\right) \rightarrow(u, v) \Longleftrightarrow u_{h} \rightarrow u \text { in } L^{s}\left(\Omega, \mathbb{R}^{n}\right), \quad v_{h} \rightarrow v \text { in measure. }
$$

The subspace $Y(\Omega)$ will be:

$$
Y(\Omega):=R_{n}(\Omega) \times B(\Omega) \subset X(\Omega),
$$

endowed with the same topology.

The convergence (3.4) is clearly metrizable. We also introduce two subspaces of $X(\Omega)$ and $Y(\Omega)$ where the trace is fixed in a strong sense:

Definition 3.4. Given $U \ni \Omega$ open and $\phi \in L^{s}(U)$ we let

$$
\begin{gathered}
X^{\phi}=\{(u, v) \in X(U): u=\phi \text { in } U \backslash \Omega\}, \\
Y^{\phi}=\{(u, v) \in Y(U): u=\phi \text { in } U \backslash \Omega\} .
\end{gathered}
$$

Following [12,13,33], we introduce a Modica-Mortola type energy to approximate the size term $\mathbf{S}\left(T_{u}\right)=$ $\mathscr{H}^{m-n}\left(S_{u} \cap \Omega\right)$. Observe that the parameter $\varepsilon$ is present with suitable exponents in order for the energy to concentrate on $(m-n)$-dimensional sets: in particular it concentrates on points if $m=n$.

Definition 3.5. Let $W \in C^{1}(\mathbb{R})$ be a nonnegative convex potential vanishing only at 0 and let $q>n$ be a given exponent. If $v \in B(\Omega)$ we set

$$
M M_{\varepsilon}(v, \Omega)=\int_{\Omega} \varepsilon^{q-n}|\nabla v|^{q}+\frac{W(1-v)}{\varepsilon^{n}} \mathrm{~d} x .
$$

Note in particular that $W$ is increasing in the positive real axis. We are now ready to introduce our family of energies:

Definition 3.6. Let $\gamma>1$ and $q>n$ be fixed exponents. We set, for $(u, v) \in X(\Omega)$ :

$$
E(u, v, \Omega)= \begin{cases}\int_{\Omega}|\nabla u|^{p}+\left|M_{n} \nabla u\right|^{\gamma} \mathrm{d} x+\sigma \mathscr{H}^{m-n}\left(S_{u} \cap \Omega\right) & \text { if } u \in G S B_{n} V(\Omega) \text { and } v=1, \\ +\infty & \text { otherwise, }\end{cases}
$$

and

$$
E_{\varepsilon}(u, v, \Omega)= \begin{cases}\int_{\Omega}|\nabla u|^{p}+\left(v+k_{\varepsilon}\right)\left|M_{n} \nabla u\right|^{\gamma} \mathrm{d} x+M M_{\varepsilon}(v, \Omega) & \text { for }(u, v) \in Y(\Omega), \\ +\infty & \text { otherwise, }\end{cases}
$$

where the constant $\sigma$ is defined by the minimum problem 4.1 and $k_{\varepsilon}$ is an infinitesimal faster than $\varepsilon^{\gamma}$. 
The first functional $E(u, v, \Omega)$ is clearly a trivial extension to $X(\Omega)$ of Definition 2.10, as $E(u, 1, \Omega)=E(u, \Omega)$. We fix once and for all a sequence $\varepsilon_{h}$ of positive numbers converging to zero and to simplify the notation we write $E_{h}$ instead of $E_{\varepsilon_{h}}$. We will also write

$$
\begin{aligned}
& F(u, 1, \Omega)=F(u, \Omega)=\int_{\Omega}|\nabla u|^{p}+\left|M_{n} \nabla u\right|^{\gamma} \mathrm{d} x, \\
& F_{\varepsilon}(u, v, \Omega)=\int_{\Omega}|\nabla u|^{p}+\left(v+k_{\varepsilon}\right)\left|M_{n} \nabla u\right|^{\gamma} \mathrm{d} x
\end{aligned}
$$

for the part of the energy explicitly depending on $u$.

We can now state our main Theorem: we prefer to present separately the lower and upper limit part of the $\Gamma$-convergence, since it is more clear where the hypotheses are used.

Theorem 3.7. Let $\Omega$ be a bounded open subset of class $C^{1}$ of $\mathbb{R}^{m}$ and suppose (2.1) and

$$
s \geq \frac{n p}{n-p}, \quad 1<\gamma \leq \frac{1}{\frac{n-1}{p}+\frac{1}{s}}, \quad q>n .
$$

(a) For every sequence $\left(\left(u_{h}, v_{h}\right)\right) \subset Y(\Omega)$ such that $\left(u_{h}, v_{h}\right) \rightarrow(u, v)$ in $X(\Omega)$ we have

$$
\liminf _{h \rightarrow \infty} E_{h}\left(u_{h}, v_{h}, \Omega\right) \geq E(u, v, \Omega)
$$

moreover

$$
\liminf _{h \rightarrow \infty} E_{h}\left(u_{h}, v_{h}, \Omega\right)<\infty \quad \Rightarrow \quad u \in G S B_{n} V(\Omega) \text { and } v=1 .
$$

(b) For every $u \in G S B_{n} V(\Omega)$ such that $E(u, 1, \Omega)<\infty$ and $\mathcal{M}^{* m-n}\left(S_{u}\right)=\mathscr{H}^{m-n}\left(S_{u}\right)$, there exists a sequence $\left(\left(u_{h}, v_{h}\right)\right) \subset Y(\Omega)$ such that $\left(u_{h}, v_{h}\right) \rightarrow(u, 1)$ in $X(\Omega)$ and

$$
\limsup _{h \rightarrow \infty} E_{h}\left(u_{h}, v_{h}, \Omega\right) \leq E(u, 1, \Omega) .
$$

Note that in particular the restrictions of $E_{h}$ and $E$ to the subspace

$$
Z(\Omega)=\left\{u \in G S B_{n} V(\Omega): \mathcal{M}_{\Omega}^{* m-n}\left(S_{u}\right)=\mathscr{H}^{m-n}\left(S_{u}\right)\right\} \times B(\Omega)
$$

satisfy (with the convergence (3.4))

$$
\Gamma-\left.\lim _{h} E_{h}\right|_{Z(\Omega)}=\left.E\right|_{Z(\Omega)} .
$$

We start the analysis on the whole family of energies $\left(E_{h}\right)$ by proving that at a fixed positive scale $\varepsilon_{h}$ the functional $E_{h}$ has a minimizer in $Y(\Omega)$, once we assign suitable Dirichlet or Neumann boundary conditions.

Theorem 3.8. Let $C \geq 0$ and $h \in \mathbb{N}$ be fixed. The sets

$$
\left\{(u, v) \in Y(U): u=\phi \text { in } U \backslash \Omega, E_{h}(u, v, U) \leq C\right\} ;
$$

with $U$ a neighborhood of $\bar{\Omega}, p^{*}>s$ and $\phi \in G S B_{n} V(\Omega)$; and

$$
\left\{(u, v) \in Y(\Omega): E_{h}(u, v, \Omega)+\int_{\Omega}|u-g|^{r} \mathrm{~d} x \leq C\right\}
$$

with $g \in L^{r}\left(\Omega, \mathbb{R}^{n}\right)$ and $r>s$, are compact subsets of $X(\Omega)$. 
Proof. Recall that it is sufficient to check sequential compactness, since (3.5) and (3.6) are subsets of the metric space $X(\Omega)$. As the product of two precompact spaces is precompact, we can examine separately the bounds on $u$ and $v$ :

$$
\int_{U}|\nabla u|^{p} \mathrm{~d} x \leq C, \quad M M_{h}(v, \Omega) \leq C .
$$

Concerning $u$ the gradients $\nabla u$ are bounded in $L^{p}$, and since

$$
\|\nabla u-\nabla \phi\|_{L^{p}\left(U, \mathbb{R}^{n \times m}\right)} \quad \text { and } \quad\|u-\phi\|_{W^{1, p}\left(U, \mathbb{R}^{n}\right)}
$$

are equivalent, by Sobolev embedding the set of $u-\phi$ 's is precompact in $L^{s}$, and so is the set of $u$ 's since $\phi \in L^{s}$. Similarly in the Neumann problem the $L^{p}$ gradient bound and the $L^{r}$ bound on $u$ give precompactness in every Lebesgue space of exponent strictly smaller than $\max \left\{r, p^{*}\right\}$, in particular in $L^{s}$. Clearly the constraint $u=\phi$ outside $\Omega$ in (3.5) is preserved. To get compactness for $v$ we can apply Young's inequality $a b \leq \frac{a^{s}}{s}+\frac{b^{t}}{t}$ with $s=\frac{q}{n}$ and $t=\frac{q}{q-n}$ to the two integrand addenda:

$$
\begin{aligned}
M M_{h}(v, \Omega) & =\int_{\Omega} \varepsilon_{h}^{q-n}|\nabla v|^{q}+\frac{W(1-v)}{\varepsilon_{h}^{n}} \mathrm{~d} x \geq \\
& \geq \int_{\Omega}\left(\frac{q}{n} \varepsilon_{h}^{q-n}|\nabla v|^{q}\right)^{\frac{n}{q}}\left(\frac{q}{q-n} \varepsilon_{h}^{-n} W(1-v)\right)^{\frac{q-n}{q}} \mathrm{~d} x \\
& =c_{n, q} \int_{\Omega}|\nabla v|^{n} W(1-v)^{\frac{q-n}{q}} \mathrm{~d} x=c_{n, q}^{\prime} \int_{\Omega}|\nabla[F(1-v)]|^{n} \mathrm{~d} x,
\end{aligned}
$$

with $F(t)=\int_{0}^{t} W^{\frac{q-n}{q n}}(s)$ d $s$. Since $\Omega$ is bounded and $0 \leq v \leq 1$ we can use the compact embedding $W^{1, n}(\Omega) \hookrightarrow$ $L^{n}(\Omega)$ to deduce that the set of $F\left(1-v_{h}\right)$ 's is precompact in $L^{n}$, hence the set of $v$ 's is precompact for the convergence in measure topology since $F$ has a continuous inverse. It remains to prove the closedness of (3.5) and (3.6): this is equivalent to show the respective energies being lower semicontinuous. Suppose then $\left(\left(u_{i}, v_{i}\right)\right) \subset X(\Omega)$ a convergent sequence and $h$ fixed. The phase transition term $M_{h}$ is clearly lower semicontinuous (see the proof of Prop. 4.1); so are also $\int_{\Omega}|\nabla u|^{p}$ and $\int_{\Omega}|u-g|^{r}$. Moreover since $k_{h}>0$

$$
\int_{\Omega}\left|M_{n} \nabla u_{i}\right|^{\gamma} \mathrm{d} x \leq \frac{C}{k_{h}}<\infty
$$

therefore up to subsequences we have $J u_{i} \stackrel{*}{\rightarrow} J u$, and by Theorem $2.8 J u \ll \mathscr{L}^{m}$, thus $u \in R_{n}(\Omega)$. Furthermore $M_{n} \nabla u_{i} \rightarrow M_{n} \nabla u$ weakly in $L^{1}$ : we claim that

$$
\int_{\Omega}\left(v(x)+k_{h}\right)\left|M_{n} \nabla u(x)\right|^{\gamma} \mathrm{d} x \leq \liminf _{i} \int_{\Omega}\left(v_{i}(x)+k_{h}\right)\left|M_{n} \nabla u_{i}(x)\right|^{\gamma} \mathrm{d} x .
$$

In fact following [29], Theorem 4.4, since $v_{i} \rightarrow v$ in measure for every $\delta>0$ there exists $G \Subset \Omega$ compact such that $v_{i} \rightarrow v$ uniformly in $G, v$ and $M_{n} \nabla u$ are continuous in $G$ and $\int_{G}\left(v+k_{h}\right)\left|M_{n} \nabla u\right|^{\gamma} \mathrm{d} x \geq \int_{\Omega}\left(v+k_{h}\right)\left|M_{n} \nabla u\right|^{\gamma} \mathrm{d} x-\delta$.

Therefore

$$
\begin{aligned}
\liminf _{i} \int_{\Omega}\left(v_{i}(x)+k_{h}\right) \mid & \left.M_{n} \nabla u_{i}(x)\right|^{\gamma} \mathrm{d} x \geq \liminf _{i} \int_{G}\left(v_{i}+k_{h}\right)\left|M_{n} \nabla u\right|^{\gamma} \mathrm{d} x \\
& +\liminf _{i} \int_{G} \gamma\left(v+k_{h}\right)\left|M_{n} \nabla u\right|^{\gamma-2}\left\langle M_{n} \nabla u, M_{n} \nabla u_{h}-M_{n} \nabla u\right\rangle \mathrm{d} x \\
& \quad+\liminf _{i} \int_{G} \gamma\left(v_{i}-v\right)\left|M_{n} \nabla u\right|^{\gamma-2}\left\langle M_{n} \nabla u, M_{n} \nabla u_{h}-M_{n} \nabla u\right\rangle \mathrm{d} x:
\end{aligned}
$$


The first integral tends to $\int_{G}\left(v+k_{h}\right)\left|M_{n} \nabla u\right|^{\gamma} \mathrm{d} x$ by uniform convergence; the second integral is infinitesimal by weak convergence, the term $\gamma\left(v+k_{h}\right)\left|M_{n} \nabla u\right|^{\gamma-2} M_{n} \nabla u$ being bounded; finally the last addendum can be bounded by

$$
\gamma\left\|M_{n} \nabla u_{h}-M_{n} \nabla u\right\|_{L^{1}(\Omega)}\left\|M_{n} \nabla u\right\|_{L^{\infty}(G)}^{\gamma-1} \sup _{G}\left|v_{i}-v\right|
$$

which is infinitesimal by uniform convergence. Therefore we can bound below the lower limit with $\int_{\Omega}(v+$ $\left.k_{h}\right)\left|M_{n} \nabla u\right|^{\gamma} \mathrm{d} x-\delta$ : letting $\delta \downarrow 0$ we obtain the claimed property.

In particular the previous Theorem guarantees that the energies $\left(E_{h}\right)$ are equicoercive, because by (3.7) the compactness of the set of $v$ 's is obtained independently of $h$. As a consequence the functionals satisfy condition (b) of Proposition 3.1, validating the choice of the topology 3.3 in the $\Gamma$-limit.

\section{Optimal Profile}

In order to investigate the asymptotic behaviour of the functionals $E_{\varepsilon}$ it is useful to understand the behaviour of the Modica-Mortola term, to single out the optimal profile and to study its properties. We consider the fixed scale $\varepsilon=1$.

Proposition 4.1. We define, for $f \in W_{\mathrm{loc}}^{1, q}\left(\mathbb{R}^{n}\right)$,

$$
I(f)=\int_{\mathbb{R}^{n}}|\nabla f|^{q}+W(f) \mathrm{d} x
$$

The infimum

$$
\sigma=\inf \{I(f) \mid I(f)<\infty, f(0)=1\}
$$

is meaningful, positive and attained by a unique radial function $w_{0} \in B\left(\mathbb{R}^{n}\right) \cap C^{0, \alpha}\left(\mathbb{R}^{n}\right)$, with $\alpha=1-\frac{n}{q}$, satisfying:

$$
\lim _{x \rightarrow \infty} w_{0}(x)=0 .
$$

Proof. First of all it is important to specify that we implicitly set $I(f)=\infty$ whenever $f$ does not possess weak derivatives in $L_{\text {loc }}^{1}$; moreover since $q>n$ the constraint requirement $f(0)=1$ in the minimization problem is meaningful, because the Sobolev embedding Theorem (see [2], 4.12) ensures that a function $f$ with $I(f)<\infty$ has a pointwise continuous representative. We will always consider the continuous representative, without specifying it anymore. Observe furthermore that since $W$ is increasing in $\mathbb{R}^{+}$and nonnegative, by truncation we can reduce to minimize the energy among functions in $B\left(\mathbb{R}^{n}\right)$ which are ranging in the interval $[0,1]$. Take a minimizing sequence $\left(f_{h}\right)$ : again by Sobolev embedding Theorem the functions $\left(f_{h}\right)$ are uniformly Hölder continuous, and equibounded on every compact subset thanks to the constraint $f_{h}(0)=1$. Hence by the Ascoli-Arzelà Theorem the sequence is precompact in the topology of the local uniform convergence, and we can extract a subsequence converging to $w_{0} \in C^{0, \alpha}$ locally uniformly. Hence $w_{0}(0)=1, W\left(f_{h}\right) \rightarrow W\left(w_{0}\right)$ locally uniformly and it is not difficult to check that $\nabla f_{h} \rightarrow \nabla w_{0}$ in $L_{\text {loc }}^{q}$. By lower semicontinuity $w_{0}$ achieves the infimum. Moreover a radial monotone rearrangement decreases the energy (see [32,41]) and by the strict convexity of the gradient part there is only one minimizer, $w_{0}$, and it is radial. Hölder continuity forces $w_{0}$ to be positive on a small ball around 0 implying that the minimum energy $\sigma$ is strictly positive; for the same reason, since $\int W\left(w_{0}\right)<\infty$, equation (4.2) must be satisfied.

Observe that $I(f)=M M_{1}\left(1-f, \mathbb{R}^{n}\right)$ for $f \in B\left(\mathbb{R}^{n}\right)$. As our optimal function $w_{0}$ is radial it is worth investigating its one dimensional profile. Setting $w:[0, \infty) \rightarrow \mathbb{R}, w(|x|)=w_{0}(x)$ we have:

$$
\sigma=\int_{\mathbb{R}^{n}}\left|\nabla w_{0}\right|^{q}+W\left(w_{0}\right) \mathrm{d} x=\mathscr{H}^{n-1}\left(S^{n-1}\right) \int_{0}^{\infty} t^{n-1}\left[\left|w^{\prime}(t)\right|^{q}+W(w(t))\right] \mathrm{d} t,
$$


and the Euler-Lagrange equation in $\mathbb{R}^{n} \backslash\{0\}$ is

$$
-q \Delta_{q} w_{0}+W^{\prime}\left(w_{0}\right):=-q \operatorname{div}\left(\left|\nabla w_{0}\right|^{q-2} \nabla w_{0}\right)+W^{\prime}\left(w_{0}\right)=0 .
$$

In radial coordinates it becomes

$$
-\frac{q}{t^{n-1}}\left(t^{n-1}\left|w^{\prime}(t)\right|^{q-2} w^{\prime}(t)\right)^{\prime}+W^{\prime}(w)=0
$$

outside the origin. We have the following Lemma:

Lemma 4.2. Let $w:[0, \infty) \rightarrow \mathbb{R}$ be the profile of the minimizer of (4.1). Then $w$ is convex, belongs to $C^{1}(0,+\infty) \cap C^{2}(\{0<w<1\})$ and the following two properties hold:

$$
\begin{gathered}
\lim _{t \rightarrow 0} t^{n}\left|w^{\prime}(t)\right|^{q}=0, \\
\lim _{t \rightarrow+\infty} t^{n}\left[\left|w^{\prime}(t)\right|^{q}+W(w(t))\right]=0 .
\end{gathered}
$$

Proof. Since $w$ is nonnegative and decreasing, and $W^{\prime} \geq 0$ by convexity, the Euler equation implies that

$$
0 \leq t^{n-1} W^{\prime}(w)=q\left(t^{n-1}\left|w^{\prime}(t)\right|^{q-2} w^{\prime}(t)\right)^{\prime}=-q\left(t^{n-1}\left|w^{\prime}(t)\right|^{q-1}\right)^{\prime} .
$$

Both the functions $t^{n-1}\left|w^{\prime}(t)\right|^{q-1}$ and $\frac{1}{t^{n-1}}$ are positive and decreasing. Hence multiplying them we get that $\left|w^{\prime}\right|$ decreases, and since $w^{\prime}$ is negative we obtain that $w$ is convex. By monotonicity of $\left|w^{\prime}\right|$ and the finiteness of the energy (4.3),

$$
\limsup _{t \rightarrow 0} t^{n}\left|w^{\prime}(t)\right|^{q} \leq \limsup _{t \rightarrow 0} n \int_{0}^{t} s^{n-1}\left|w^{\prime}(s)\right|^{q} \mathrm{~d} s=0 .
$$

Furthermore, since $Z(t):=\left|w^{\prime}(t)\right|^{q}+W(w(t))$ is decreasing, we have

$$
\limsup _{t \rightarrow \infty} \frac{1}{n}\left(1-\frac{1}{2^{n}}\right) t^{n} Z(t) \leq \limsup _{t \rightarrow \infty} \int_{\frac{t}{2}}^{t} s^{n-1} Z(s) \mathrm{d} s \leq \limsup _{t \rightarrow \infty} \int_{\frac{t}{2}}^{\infty} s^{n-1} Z(s) \mathrm{d} s=0
$$

by the finiteness of the energy (4.3), which proves (4.6). Finally in every interval $(a, b) \Subset\{0<w<1\}$ we have that $-\infty<w^{\prime}<w^{\prime}(b)<0$, otherwise $w$ would be a positive constant in the half line $(b,+\infty)$. Hence we can extract the $(q-1)$-st root without loosing any smoothness and bootstrap (4.4):

$$
w \in C^{0, \alpha}(a, b) \quad \Rightarrow \quad W^{\prime}(w) \in C^{0}(a, b) \quad \Rightarrow \quad w \in C^{2}(a, b) .
$$

The same argument shows that $w \in C^{1}(0,+\infty)$, since $|\cdot|^{\frac{1}{q-1}}$ is continuous.

In general if $W \in C^{k}$ and $w \in C^{m}(a, b)$ then $W^{\prime}(w) \in C^{m \wedge(k-1)}(a, b)$, which gives $w \in C^{(m \wedge(k-1))+2}(a, b)$ : therefore starting from $m=1$ we obtain $w \in C^{k+1}(\{0<w<1\})$.

\section{5. $\Gamma$-LOWER LIMIT}

In this section we aim to prove the first part of Theorem 3.7, regarding the $\Gamma$-lower limit of the sequence $\left(E_{h}\right)$ :

Theorem 5.1. Let $\Omega$ be an open subset of $\mathbb{R}^{m}$. For every sequence $\left(\left(u_{h}, v_{h}\right)\right) \subset Y(\Omega)$ such that $\left(u_{h}, v_{h}\right) \rightarrow(u, v)$ we have

moreover

$$
\liminf _{h \rightarrow \infty} E_{h}\left(u_{h}, v_{h}, \Omega\right) \geq E(u, v, \Omega)
$$

$$
\liminf _{h \rightarrow \infty} E_{h}\left(u_{h}, v_{h}, \Omega\right)<\infty \quad \Rightarrow \quad u \in G S B_{n} V(\Omega) \text { and } v=1 .
$$

The proof will be achieved through a slicing argument, by first proving that in codimension $m-n=0$ the jacobians $J u_{h}$ concentrate around a finite number of points. Our definition of size outlined in the introduction is well-suited to this slicing procedure, and a final localization result yields the proof. 


\subsection{Proof in $\mathbb{R}^{n}$}

Let $A$ be an open subset of $\mathbb{R}^{n}$ : to ease the exposition for any $(u, v) \in Y(A)$ we let

$$
G_{h}(u, v, A)=\int_{A}\left(v+k_{h}\right)|\operatorname{det} \nabla u|^{\gamma} \mathrm{d} x+\int_{A} \varepsilon_{h}^{q-n}|\nabla v|^{q}+\frac{W(1-v)}{\varepsilon_{h}^{n}} \mathrm{~d} x
$$

be the part of energy depending explicitly on $v$.

Theorem 5.2. Let $A$ be an open subset of $\mathbb{R}^{n}$ and let $\left(\left(u_{h}, v_{h}\right)\right) \subset Y(A),(u, v) \in X(\Omega)$ satisfy $\left(u_{h}, v_{h}\right) \rightarrow(u, v)$ and $\left\|\nabla u_{h}\right\|_{p} \leq C$. Assume also

$$
\liminf _{h \rightarrow \infty} G_{h}\left(u_{h}, v_{h}, A\right)<\infty .
$$

Then $u \in G S B_{n} V\left(A, \mathbb{R}^{n}\right), v=1$ and

$$
\liminf _{h \rightarrow \infty} G_{h}\left(u_{h}, v_{h}, A\right) \geq \int_{A}|\operatorname{det} \nabla u|^{\gamma} \mathrm{d} x+\sigma \mathscr{H}^{0}\left(A \cap S_{u}\right) .
$$

First of all we extract a subsequence, not relabeled, that achieves the lower limit in (5.1) and such that $\nabla u_{h} \rightarrow$ $\nabla u$ weakly in $L^{p}$. We notice right away that $u \in W^{1, p}$ and $v=1$; also by $(2.4)$ we know that $\mathbf{F}\left(J u_{h}-J u\right) \rightarrow 0$, hence $J u_{h} \stackrel{*}{\rightarrow} J u$ as currents. We begin with the regular part, disregarding the positive infinitesimal $k_{h}$ :

Lemma 5.3. Assume that $A$ is a bounded open subset of $\mathbb{R}^{n}$ with Lipschitz boundary. Then

$$
\liminf _{h \rightarrow \infty} \int_{A} v_{h}\left|\operatorname{det} \nabla u_{h}\right|^{\gamma} \mathrm{d} x \geq \int_{A}|\operatorname{det} \nabla u|^{\gamma} \mathrm{d} x .
$$

Proof. Since $A$ is regular and bounded, $q>n$ and the norms $\left\|\nabla v_{h}\right\|_{q}$ are equibounded by Sobolev embedding Theorem

$$
\left[v_{h}\right]_{C^{\alpha}(A)} \leq C(A) \varepsilon_{h}^{-\alpha},
$$

where $\alpha=1-\frac{n}{q}$ and $C(A)$ depends on the energy and on the regularity of $A$. We also fix a threshold $t \in(0,1)$ : by Hölder continuity there exists $c_{0}=c_{0}(C, t)>0$ independent of $h$ such that for every $x \in A \cap\left\{v_{h}<t\right\}$

$$
A \cap B\left(x, c_{0} \varepsilon_{h}\right) \subset A \cap\left\{v_{h}<\frac{1+t}{2}\right\} .
$$

We can then cover $A \cap\left\{v_{h}<t\right\}$ with balls centered at every point having radius $\frac{c_{0} \varepsilon_{h}}{5}$ : by Vitali's covering Lemma there is a countable disjoint subfamily $\mathcal{F}=\left\{B\left(x_{i}, \frac{c_{0} \varepsilon_{h}}{5}\right)\right\}$ such that

$$
\bigcup_{i} B\left(x_{i}, c_{0} \varepsilon_{h}\right) \supset A \cap\left\{v_{h}<t\right\} .
$$

Thanks to (5.3) we can estimate from below $M M_{\varepsilon_{h}}$ of every such small ball:

$$
\int_{A \cap B\left(x_{i}, \frac{c_{0} \varepsilon_{h}}{5}\right)} \varepsilon_{h}^{q-n}\left|\nabla v_{h}\right|^{q}+\frac{W\left(1-v_{h}\right)}{\varepsilon_{h}^{n}} \mathrm{~d} x \geq W\left(\frac{1-t}{2}\right) \frac{\mathscr{L}^{n}\left(A \cap B\left(x_{i}, \frac{c_{0} \varepsilon_{h}}{5}\right)\right)}{\varepsilon_{h}^{n}} .
$$

The latter quantity is bounded below independently of $h$ because the Lipschitz boundary condition on $A$ ensures that $\mathscr{L}^{n}\left(A \cap B\left(x_{i}, \frac{c_{0} \varepsilon_{h}}{5}\right)\right) \geq c \varepsilon_{h}^{n}$. The family $\mathcal{F}$ being disjoint, by the finiteness of the energy we argue that there can be only a finite number $N$, independent of $h$, of such balls. Let us then extract a subsequence, not relabeled, along which the balls are in constant number $N$ and the centers $\left\{x_{i}^{h}\right\}, i=1, \ldots, N$ converge to points $x_{i}^{\infty} \in \bar{A}$. For every open set

$$
A^{\prime} \Subset A \backslash \bigcup_{i}\left\{x_{i}^{\infty}\right\}
$$


we have that for $h$ sufficiently large:

$$
A^{\prime} \cap \bigcup_{i} B\left(x_{i}^{h}, c_{0} \varepsilon_{h}\right)=\emptyset \quad \text { and }\left.\quad v_{h}\right|_{A^{\prime}} \geq t .
$$

The energy bound (5.1) allows to bound a superlinear power of the jacobians in $A^{\prime}$

$$
\int_{A^{\prime}}\left|\operatorname{det} \nabla u_{h}\right|^{\gamma} \mathrm{d} x \leq \frac{C}{t+1}
$$

hence Theorem 2.8 gives

$$
\operatorname{det} \nabla u_{h} \rightarrow \operatorname{det} \nabla u \quad \text { weakly in } L^{1}\left(A^{\prime}\right)
$$

By lower semicontinuity

$$
\begin{aligned}
\liminf _{h \rightarrow \infty} \int_{A} v_{h}\left|\operatorname{det} \nabla u_{h}\right|^{\gamma} \mathrm{d} x & \geq \liminf _{h \rightarrow \infty} \int_{A^{\prime}} v_{h}\left|\operatorname{det} \nabla u_{h}\right|^{\gamma} \mathrm{d} x \geq \\
& \geq \liminf _{h \rightarrow \infty} t \int_{A^{\prime}}\left|\operatorname{det} \nabla u_{h}\right|^{\gamma} \mathrm{d} x \geq t \int_{A^{\prime}}|\operatorname{det} \nabla u|^{\gamma} \mathrm{d} x .
\end{aligned}
$$

Finally letting $A^{\prime} \uparrow A \backslash \bigcup_{i}\left\{x_{i}^{\infty}\right\}$ and then $t \uparrow 1$ we obtain the result.

Remark 5.4. The same result of Lemma 5.3 holds without the regularity hypothesis on $A$. In fact it is sufficient to consider a sequence of nested regular open subsets $A_{j} \subset A$ invading $A$, apply the Lemma to $A_{j}$ and then let $A_{j} \uparrow A$ : the left hand side of (5.2) clearly decreases when restricted to each $A_{j}$, and the right hand side by Monotone convergence Theorem increases to $\int_{A}|\operatorname{det} \nabla u|^{\gamma} \mathrm{d} x$.

Now we analyze the $M M_{\varepsilon}$ term, and prove that around the potentially singular points of the limit function $u$ this energy concentrates. Observe that we still do not know that $u \in G S B_{n} V: J u$ so far is only a flat current, nevertheless chosen a fixed point $x_{0}$ for almost every radius $\rho$ the restriction $J u\left\llcorner B_{\rho}\left(x_{0}\right)\right.$ is meaningful and furthermore $\mathbf{F}\left(J u_{h}\left\llcorner B_{\rho}\left(x_{0}\right)-J u\left\llcorner B_{\rho}\left(x_{0}\right)\right) \rightarrow 0\right.\right.$ (see [9], Sect. 2.2). With a slight abuse of notation we indicate by $J u\left\llcorner B_{\rho} \ll \mathscr{L}^{n}\right.$ the fact that $\mathbf{M}\left(J u\left\llcorner B_{\rho}\right)<\infty\right.$ and $\| J u\left\llcorner B_{\rho} \| \ll \mathscr{L}^{n}\right.$ : by definition this is satisfied if $u \in R_{n}$.

Lemma 5.5. Let $\left(\left(u_{h}, v_{h}\right)\right), u$ and $A$ as in Theorem 5.2, and fix $x_{0} \in A$. Suppose $J u\left\llcorner B_{\rho}\left(x_{0}\right) \nless \mathscr{L}^{n}\right.$ for every $\rho>0$ such that $B_{\rho}\left(x_{0}\right) \subset A$. Then

$$
\liminf _{h \rightarrow \infty} M M_{h}\left(v_{h}, B_{\rho}\left(x_{0}\right)\right) \geq \sigma \quad \forall \rho>0,
$$

where $\sigma$ is defined as in (4.1).

Proof. Fix an arbitrary $\rho$ as in the hypotheses and let us suppose for simplicity that $x_{0}=0$ : since $J u\left\llcorner B_{\rho} \nless \mathscr{L}^{n}\right.$ we must have

$$
\lim _{h \rightarrow \infty} \inf _{B_{\rho}} v_{h}=0 \quad \forall \rho>0 .
$$

In fact if there were a radius $\bar{\rho}$ and a subsequence $\left(v_{\bar{h}}\right)$ bounded below by some $\delta>0$ in $B_{\bar{\rho}}$, we would have the uniform bound $\sup _{\bar{h}} \int_{B_{\bar{\rho}}}\left|\operatorname{det} \nabla u_{\bar{h}}\right|^{\gamma} \mathrm{d} x \leq C \delta^{-1}$. Therefore we could apply Theorem 2.8 with $\Psi(t)=|t|^{\gamma}$ : since $J u_{\bar{h}}=\operatorname{det} \nabla u_{\bar{h}} \mathbf{E}^{m} \ll \mathscr{L}^{m}$ by the weak $L^{1}$ convergence (2.6) the limit $J u L B_{\bar{\rho}}$ would otherwise be a current in $\mathbf{M}_{0}\left(B_{\bar{\rho}}\right)$ with absolutely continuous mass. The finiteness of the energy (5.1) guarantees that $v_{h} \rightarrow 1$ in measure in $B_{\rho}$. In order to show (5.6) we modify in $B_{\rho}$ the asymptotic profiles $v_{h}$ and we relate them to problem (4.1). Let us perform the following radial monotone rearrangement of $v_{h}$, denoted $v_{h}^{*}$, which preserve the measure of sublevels:

$$
v_{h}^{*}(x):=\inf \left\{t:\left|\left\{v_{h}<t\right\} \cap B_{\rho}\right|>\mathscr{L}^{n}\left(B_{1}\right)|x|^{n}\right\} \quad \forall x \in B_{\rho} .
$$


This rearrangement preserves the integral $\int_{B_{\rho}} W(1-v)$ by the Coarea formula $(2.13)$, while the $L^{q}$ norm of the gradient decreases, see $[32,41]$. We immediately have that

$$
M M_{h}\left(v_{h}, B_{\rho}\right) \geq M M_{h}\left(v_{h}^{*}, B_{\rho}\right) \text { and } v_{h}^{*} \rightarrow 1 \text { in measure in } B_{\rho} .
$$

In particular $\lambda_{h}:=\left.v_{h}^{*}\right|_{\partial B_{\rho}} \rightarrow 1$, and $\mu_{h}:=\inf _{B_{\rho}} v_{h}^{*}=v_{h}^{*}(0) \rightarrow 0$, hence we can extend $v_{h}^{*}$ equal to $\lambda_{h}$ for $|x| \geq \rho$. The functions $f_{h}: \mathbb{R}^{n} \rightarrow \mathbb{R}$,

$$
f_{h}(y):=\frac{1}{\lambda_{h}-\mu_{h}}\left(v_{h}^{*}\left(\varepsilon_{h} y\right)-\mu_{h}\right)
$$

satisfy
(a) $f_{h}(0)=0$,
(b) $\operatorname{spt}\left(1-f_{h}\right) \subset \overline{B_{\rho}}$,
(c) $1-v_{h}^{*}(x) \geq \lambda_{h}-v_{h}^{*}(x)=\left(\lambda_{h}-\mu_{h}\right)\left(1-f_{h}\left(\frac{x}{\varepsilon_{h}}\right)\right)$.

Let us now evaluate the $M M_{h}$ energy (recall $W$ is monotone increasing):

$$
\begin{aligned}
M M_{h}\left(v_{h}, B_{\rho}\right) \geq M M_{h}\left(v_{h}^{*}, B_{\rho}\right) & =\int_{B_{\rho}} \varepsilon_{h}^{q-n}\left|\nabla v_{h}^{*}(x)\right|^{q}+\frac{W\left(1-v_{h}^{*}(x)\right)}{\varepsilon_{h}^{n}} \mathrm{~d} x \\
& \stackrel{(c)}{\geq} \int_{B_{\rho}} \varepsilon_{h}^{q-n}\left|\nabla v_{h}^{*}(x)\right|^{q}+\frac{W\left(\lambda_{h}-v_{h}^{*}(x)\right)}{\varepsilon_{h}^{n}} \mathrm{~d} x \\
& \geq \int_{\mathbb{R}^{n}}\left(\lambda_{h}-\mu_{h}\right)^{q}\left|\nabla f_{h}\right|^{q}+W\left(\left(\lambda_{h}-\mu_{h}\right)\left(1-f_{h}\right)\right) \mathrm{d} x .
\end{aligned}
$$

By properties (a) and (b) the functions $1-f_{h}$ are competitors for problem (4.1) and $\lambda_{h}-\mu_{h} \rightarrow 1$, hence the last integral is asymptotically greater or equal than the infimum $\sigma$.

Proof of Theorem 5.2. Let $\Sigma=\left\{x \in A: J u\left\llcorner B_{\rho}(x) \nless \mathscr{L}^{n}\right.\right.$ for all $\left.B_{\rho}(x) \subset A\right\}$. Then the superadditivity of the lim inf together with (5.1) and Lemma 5.5 gives

$$
\mathscr{H}^{0}(\Sigma) \leq \frac{1}{\sigma} \liminf _{h} G_{h}\left(u_{h}, v_{h}, A\right) .
$$

Moreover Lemma 5.3 showed the existence of another finite set $\Upsilon$ such that $J u\left\llcorner(A \backslash \Upsilon) \ll \mathscr{L}^{n}\right.$. Hence necessarily $\Sigma \subset \Upsilon$ and the flat defect current

$$
T:=\left(J u-\operatorname{det} \nabla u \mathbf{E}^{m}\right)\llcorner A
$$

is supported in $\Upsilon$. By the general theory of flat currents (see [9], Thm. 3.4, [24], 4.1.18) $\mathbf{M}(T)<\infty$ and $T=\sum_{x_{i} \in \Sigma} a_{i} \llbracket x_{i} \rrbracket$. In particular $u \in G S B_{n} V(A)$ and $S_{u} \subset \Sigma$, so

$$
\mathscr{H}^{0}\left(S_{u} \cap A\right) \leq \mathscr{H}^{0}(\Sigma)
$$

Taking $B \Subset A \backslash \Sigma$ open, and applying the superadditivity of the lower limit on open disjoint sets, as well as (5.2) to $B$ we obtain for some $\rho$ sufficiently small (so that $B \cap \bigcup_{x \in \Sigma} B_{\rho}(x)=\emptyset$ )

$$
\begin{aligned}
\liminf _{h} G_{h}\left(u_{h}, v_{h}, A\right) & \geq \liminf _{h} \int_{B} v_{h}\left|\operatorname{det} \nabla u_{h}\right|^{\gamma} \mathrm{d} x \\
& +\sum_{x \in S_{u} \cap A} \liminf _{h} \int_{B_{\rho}(x) \cap A} \varepsilon_{h}^{q-2}\left|\nabla v_{h}\right|^{q}+\frac{W\left(1-v_{h}\right)}{\varepsilon_{h}^{2}} \mathrm{~d} x \\
& \geq \int_{B}|\operatorname{det} \nabla u|^{\gamma} \mathrm{d} x+\sigma \mathscr{H}^{0}\left(S_{u} \cap A\right) .
\end{aligned}
$$

Letting $B \uparrow A$ concludes the proof. 


\subsection{Reduction argument and proof of Theorem 5.1 for general $m, n$}

In this paragraph we prove Theorem 5.1 from the results obtained in the previous paragraph in dimension $n$. We will first use the slicing properties of the jacobians to reduce to the $n$-dimensional case discussed above, and then we will optimize the choices of the slicing directions to conclude.

Proof. As a preliminary step let us extract a subsequence out of $\left(\left(u_{h}, v_{h}\right)\right)$ such that the lower limit $\liminf _{h} E_{h}\left(u_{h}, v_{h}, \Omega\right)$ is attained and such that $\left(u_{h}, v_{h}\right) \rightarrow(u, 1)$ rapidly in $X(\Omega)$ :

$$
\sum_{h}\left\|u_{h}-u\right\|_{L^{s}}+d\left(v_{h}, 1\right)<\infty .
$$

This implies that given an orthogonal projection $\pi \in \mathbf{O}_{m-n}$, for $\mathscr{L}^{m-n}$-almost every $x \in \pi(\Omega)$

$$
u_{h}(x, \cdot) \rightarrow u(x, \cdot) \text { in } L^{s}\left(\Omega^{x}, \mathbb{R}^{n}\right) \quad \text { and } \quad v_{h}(x, \cdot) \rightarrow 1 \text { in measure in } \Omega^{x},
$$

where we put $\Omega^{x}:=\Omega \cap \pi^{-1}(x)$. Let us consider an arbitrary open subset $A \subset \Omega$ and let us fix a projection $\pi$ as above. Observe that the energy $E_{h}$ is bounded along $\left(u_{h}, v_{h}\right)$ : using Fatou's Lemma we obtain

$$
\begin{aligned}
& \lim _{h \rightarrow \infty} E_{h}\left(u_{h}, v_{h}, \Omega\right) \geq \liminf _{h \rightarrow \infty} E_{h}\left(u_{h}, v_{h}, A\right) \geq \\
& \quad \geq \int_{\pi(A)} \liminf _{h \rightarrow \infty}\left\{\int_{A^{x}}\left|\nabla_{y} u_{h}\right|^{p}+v_{h}\left|\operatorname{det} \nabla_{y} u_{h}\right|^{\gamma}+\varepsilon_{h}^{q-n}\left|\nabla_{y} v_{h}\right|^{q}+\frac{W\left(1-v_{h}\right)}{\varepsilon_{h}^{n}} \mathrm{~d} y\right\} \mathrm{d} x .
\end{aligned}
$$

In particular for $\mathscr{L}^{m-n}$-almost every $x \in \pi(A)$

$$
\liminf _{h} \int_{A^{x}}\left|\nabla_{y} u_{h}(x, y)\right|^{p} \mathrm{~d} y+G_{h}\left(u_{h}, v_{h}, A^{x}\right) \leq C(x)<\infty .
$$

For these $x$ we can extract a subsequence $\left(u_{h(k)}\right)$, a priori depending on the point $x$, along which both the $L^{p}$ norm of $\nabla u_{h(k)}$ and the $n$-dimensional energy $G_{h}$ are bounded:

$$
\sup _{k} \int_{A^{x}}\left|\nabla_{y} u_{h(k)}\right|^{p} \mathrm{~d} y+G_{h}\left(u_{h(k)}, v_{h(k)}, A^{x}\right)<\infty .
$$

This implies that

$$
\nabla_{y} u_{h(k)}(x, \cdot) \rightarrow \nabla_{y} u(x, \cdot) \text { in } L^{p}\left(A^{x}\right) .
$$

Finally observe that the slicing Theorem 2.3 immediately gives that $\left(u_{h}(x, \cdot), v_{h}(x, \cdot)\right) \in Y\left(A^{x}\right)$ almost everywhere. Theorem 5.2 implies that $u(x, \cdot) \in G S B_{n} V\left(A^{x}\right)$ and that

$$
\liminf _{h} G_{h}\left(u_{h}, v_{h}, A^{x}\right) \geq \int_{A^{x}}\left|\operatorname{det} \nabla_{y} u(x, \cdot)\right|^{\gamma} \mathrm{d} y+\sigma \mathscr{H}^{0}\left(A^{x} \cap S_{u(x, \cdot)}\right) ;
$$

integrating and applying Fatou's Lemma on the left hand side we have

$$
\begin{aligned}
\liminf _{h} E_{h}\left(u_{h}, v_{h}, A\right) & \geq \int_{A}|\nabla u|^{p}+\int_{\pi(A)} \liminf _{h} G_{h}\left(u_{h}(x, \cdot), v_{h}(x, \cdot), A^{x}\right) \mathrm{d} x \geq \\
& \geq \int_{A}|\nabla u|^{p}+\int_{A}\left|\operatorname{det} \nabla_{y} u(x, \cdot)\right|^{\gamma} \mathrm{d} y \mathrm{~d} x+\sigma \int_{\pi(A)} \mathscr{H}^{0}\left(A^{x} \cap S_{u(x, \cdot)}\right) \mathrm{d} x .
\end{aligned}
$$

Let us call

$$
\tau_{\pi}(A):=\int_{A}\left|\operatorname{det} \nabla_{y} u(x, y)\right|^{\gamma} \mathrm{d} y \mathrm{~d} x+\sigma \int_{\pi(A)} \mathscr{H}^{0}\left(A^{x} \cap S_{u(x, \cdot)}\right) \mathrm{d} x .
$$


the right hand side and $\underline{E}(A)=\liminf _{h} E_{h}\left(u_{h}, v_{h}, A\right) \cdot \underline{E}(\cdot)$ is a superadditive set function on open sets such that $\underline{E}(A) \leq \underline{E}(\Omega)<\infty$ and each single $\tau_{\pi}$ is a finite Borel measure; therefore taking disjoint open sets $A_{1}, \ldots, A_{k}$ and orthogonal projections $\pi_{1}, \ldots, \pi_{k}$ we have that

$$
\sum_{i} \tau_{\pi_{i}}\left(A_{i}\right) \leq \sum_{i} \underline{E}\left(A_{i}\right) \leq \underline{E}(\Omega) .
$$

By inner and outer regularity of $\tau_{\pi_{i}}$ inequality (5.11) holds for generic disjoint Borel sets $B_{i}$ instead of $A_{i}$, hence the supremum

$$
\tau:=\bigvee_{\pi} \tau_{\pi}
$$

is a finite Borel measure. In particular $M_{n} \nabla u \in L^{\gamma}$ and since for every projection $\pi$ slice and jacobian commute according to Theorem 2.3, we have that the current $T=\left(J u-M_{n} \nabla u \mathbf{E}^{m}\right)\left\llcorner\Omega\right.$ satisfies $\operatorname{spt}(\langle T, \pi, x\rangle) \subset S_{u(x, \cdot)}$ almost everywhere, so its size is finite. Hence $u \in G S B_{n} V(\Omega)$. Finally since by Definition 2.4 the measures $\mu_{T}$ and $\left|M_{n} \nabla u\right|^{\gamma} \mathscr{L}^{m}$ are mutually singular and $\left|M_{n} L\right|=\sup _{\pi}\left|M_{n} L L d \pi\right|$, it is not difficult to prove that the supremum $\tau$ equals

$$
\tau=\left|M_{n} \nabla u\right|^{\gamma} \mathscr{L}^{m}+\sigma \mathscr{H}^{m-n}\left\llcorner S_{u},\right.
$$

which concludes the proof.

\section{6. $\Gamma$-UPPER LIMIT}

This section is devoted to the proof of the upper limit inequality: our construction of the recovery sequence will mimic the truncation argument presented in $[12,13]$. Note that we only assume a mild geometric property on the singular set $S_{u}$ expressed in terms of its Minkowski content. We provide an interior statement as well as boundary statement, where differently from [12] we need to take care of any possible accumulation of the singular set at the boundary. The limit energy must account for the possible loss of mass in the Modica-Mortola term, due to the transition of $v$ happening partially outside the domain. We finally generalize the form of the functional in which the size term is weighted by a continuous density.

Theorem 6.1. Suppose $\Omega \subset \mathbb{R}^{m}$ is a bounded set of class $C^{1}$ and $u \in G S B_{n} V(\Omega)$ with constraints

$$
s \geq \frac{n p}{n-p}, \quad 1<\gamma \leq \frac{1}{\frac{n-1}{p}+\frac{1}{s}} .
$$

Let also $\left(k_{h}\right)$ be a positive sequence such that $k_{h}=o\left(\varepsilon_{h}^{\gamma}\right)$. If

$$
E(u, 1, \Omega)<\infty, \quad \mathcal{M}_{\Omega}^{* m-n}\left(S_{u}\right)=\mathscr{H}^{m-n}\left(S_{u}\right)
$$

then there exists a sequence $\left(\left(u_{h}, v_{h}\right)\right) \subset Y(\Omega)$ such that

$$
\left(u_{h}, v_{h}\right) \rightarrow(u, 1) \quad \text { and } \quad \limsup _{h \rightarrow \infty} E_{h}\left(u_{h}, v_{h}, \Omega\right) \leq E(u, 1, \Omega) .
$$

\subsection{Proof of Theorem 6.1}

We start by setting the approximating sequence $\left(v_{h}\right)$ for a generic $\mathscr{L}^{m}$-null set $S \subset \Omega$ satisfying

$$
\mathcal{M}_{\Omega}^{* m-n}(S)=\mathscr{H}^{m-n}(S)<\infty .
$$

Let $w(t)$ be the optimal profile of problem 4.1 and choose $\delta_{h} \downarrow 0$ such that $k_{h}\left(\varepsilon_{h} \delta_{h}\right)^{-\gamma} \rightarrow 0$. Let

$$
w_{h}(t):=\min \left\{\frac{w(t)}{w\left(\delta_{h}\right)}, 1\right\}
$$


so that $w_{h}(|x|)=1$ in $B_{\delta_{h}}(0)$. Clearly $w_{h}^{\prime}\left(\delta_{h}\right)$ is finite and $I\left(w_{h}\right) \rightarrow I(w)$ as $h \rightarrow \infty$. Moreover by the proof of Lemma 4.2

$$
\left|w^{\prime}(t)\right|^{q}+W(w(t))
$$

is $C^{1}$ and decreases to 0 for $t \rightarrow \infty$ : these properties hold true in $\left(\delta_{h}+\infty\right)$ for $w_{h}$. Set

$$
v_{h}(x)=1-w_{h}\left(\frac{\mathrm{d}(x, S)}{\varepsilon_{h}}\right),
$$

where $d(x, S)=\operatorname{dist}(x, S):$ note that $v_{h} \rightarrow 1$ in measure and that by equation $(2.12)$

$$
\left|\nabla v_{h}(x)\right|=\frac{1}{\varepsilon_{h}}\left|w_{h}^{\prime}\left(\frac{\mathrm{d}(x, S)}{\varepsilon_{h}}\right)\right|
$$

at almost every point $x$. Recall the notation $S_{r}=\{x \in \Omega: 0<\operatorname{dist}(x, S) \leq r\}$ and $V(r)=\mathscr{L}^{m}\left(S_{r}\right)$.

Proposition 6.2. The functions $\left(v_{h}\right)$ satisfy

$$
v_{h}=0 \quad \text { on } \quad S_{\varepsilon_{h} \delta_{h}}
$$

and

$$
\limsup _{h \rightarrow \infty} M M_{h}\left(v_{h}, \Omega\right) \leq \sigma \mathcal{M}_{\Omega}^{* m-n}(S) .
$$

Proof. The first statement is true by the definition (6.3). Looking at the energy

$$
M M_{h}\left(v_{h}, \Omega\right)=\int_{\Omega} \varepsilon_{h}^{q-n}\left|\nabla v_{h}\right|^{q}+\frac{W\left(1-v_{h}\right)}{\varepsilon_{h}^{n}} \mathrm{~d} x
$$

we observe right away that the integration on the set $S_{\varepsilon_{h} \delta_{h}}$ in infinitesimal, since there $v_{h}$ is identically 0 and so

$$
\int_{S_{\varepsilon_{h} \delta_{h}}} \varepsilon_{h}^{q-n}\left|\nabla v_{h}\right|^{q}+\frac{W\left(1-v_{h}\right)}{\varepsilon_{h}^{n}} \mathrm{~d} x=W(1) \frac{V\left(\varepsilon_{h} \delta_{h}\right)}{\varepsilon_{h}^{n}} \rightarrow 0 .
$$

Applying the Coarea formula (2.13) on the level sets of the distance function $d(\cdot, S)$ we can write

$$
\begin{aligned}
M M_{h}\left(v_{h}, \Omega\right) & =o(1)+\int_{\Omega \backslash S_{\varepsilon_{h} \delta_{h}}} \varepsilon_{h}^{q-n}\left|\nabla v_{h}\right|^{q}+\frac{W\left(1-v_{h}\right)}{\varepsilon_{h}^{n}} \mathrm{~d} x \\
& =o(1)+\int_{\varepsilon_{h} \delta_{h}}^{+\infty}\left[\left|w_{h}^{\prime}\left(\frac{t}{\varepsilon_{h}}\right)\right|^{q}+W\left(w_{h}\left(\frac{t}{\varepsilon_{h}}\right)\right)\right] \frac{V^{\prime}(t)}{\varepsilon_{h}^{n}} \mathrm{~d} t \\
& =o(1)+\int_{\delta_{h}}^{+\infty}\left[\left|w_{h}^{\prime}(s)\right|^{q}+W\left(w_{h}(s)\right)\right] \frac{\left[V\left(\varepsilon_{h} s\right)\right]^{\prime}}{\varepsilon_{h}^{n}} \mathrm{~d} s .
\end{aligned}
$$

Since $Z_{h}(s):=\left|w_{h}^{\prime}(s)\right|^{q}+W\left(w_{h}(s)\right)$ is $C^{1}$ we can integrate by parts

$$
\int_{\delta_{h}}^{+\infty} Z_{h}(s) \frac{\left[V\left(\varepsilon_{h} s\right)\right]^{\prime}}{\varepsilon_{h}^{n}} \mathrm{~d} s=-\int_{\delta_{h}}^{+\infty} Z_{h}^{\prime}(s) \frac{V\left(\varepsilon_{h} s\right)}{\varepsilon_{h}^{n}} \mathrm{~d} s+\frac{Z_{h}(+\infty) V(+\infty)-Z_{h}\left(\delta_{h}\right) V\left(\varepsilon_{h} \delta_{h}\right)}{\varepsilon_{h}^{n}} .
$$

As previously outlined $Z_{h}(+\infty)=0$ and $V(+\infty)=\mathscr{L}^{m}(\Omega)$, hence the second addendum is null; moreover $\frac{V\left(\varepsilon_{h} \delta_{h}\right)}{\varepsilon_{h}^{n}} \leq\left(\mathcal{M}_{\Omega}^{* m-n}(S)+1\right) \mathscr{L}^{n}\left(B_{1}\right) \delta_{h}^{n}$ and $Z_{h}\left(\delta_{h}\right)=\left|w_{h}^{\prime}\left(\delta_{h}\right)\right|^{q}+W\left(w_{h}\left(\delta_{h}\right)\right)$, so also the third term goes to 0 by Lemma 4.2. The basic Assumption (6.2) on the Minkowski content of $S$ implies that there exist infinitesimal numbers $\xi_{h}$ such that

$$
V(s) \leq \mathscr{L}^{n}\left(B_{1}\right) \mathcal{M}_{\Omega}^{* m-n}(S) s^{n}+\xi_{h} s^{n} \quad \forall s \in\left[0, \varepsilon_{h} \operatorname{diam}(\Omega)\right]
$$


Recall that $Z_{h}^{\prime}(s) \leq 0$ in $\left[\delta_{h}, \infty\right)$ and $I\left(w_{h}\right) \rightarrow I(w)=\sigma$. Then, integrating by parts

$$
\begin{aligned}
M M_{h}\left(v_{h}, \Omega\right) & =o(1)-\int_{\delta_{h}}^{+\infty} Z_{h}^{\prime}(s) \frac{V\left(\varepsilon_{h} s\right)}{\varepsilon_{h}^{n}} \mathrm{~d} s \\
& \leq o(1)-\int_{\delta_{h}}^{+\infty} Z_{h}^{\prime}(s)\left(\mathscr{L}^{n}\left(B_{1}\right) \mathcal{M}_{\Omega}^{* m-n}(S)+\xi_{h}\right) s^{n} \mathrm{~d} s \\
& \stackrel{(4.5)(4.6)}{=} o(1)+n\left(\mathscr{L}^{n}\left(B_{1}\right) \mathcal{M}_{\Omega}^{* m-n}(S)+\xi_{h}\right) \int_{\delta_{h}}^{+\infty} s^{n-1} Z_{h}(s) \mathrm{d} s \\
& =o(1)+\left(\mathscr{H}^{n-1}\left(S^{n-1}\right) \mathcal{M}_{\Omega}^{* m-n}(S)+n \xi_{h}\right) \int_{\delta_{h}}^{+\infty} s^{n-1} Z_{h}(s) \mathrm{d} s \\
& =o(1)+\mathcal{M}_{\Omega}^{* m-n}(S) \cdot I\left(w_{h}\right)=o(1)+\sigma \mathcal{M}_{\Omega}^{* m-n}(S) .
\end{aligned}
$$

Remark 6.3. Observe that the same Proposition proves something more general, that will be useful in the sequel: if $\bar{w}$ is a radial profile such that $\bar{Z}(t):=\left|\bar{w}^{\prime}(t)\right|^{q}+W(\bar{w}(t))$ is decreasing, then the sequence $\left(v_{h}\right)$ constructed from $\bar{w}$ as in (6.3) satisfies:

$$
\limsup _{h} M M_{h}\left(v_{h}, \Omega\right) \leq I(\bar{w}(|x|)) \mathcal{M}_{\Omega}^{* m-n}(S) .
$$

We now show how to construct the sequence $\left(u_{h}\right)$. Outside $S_{\varepsilon_{h} \delta_{h}}$ the jacobian $J u$ is absolutely continuous, hence there is no need to modify $u$ there. We will only change $u$ inside $S_{\varepsilon_{h} \delta_{h}}$ with the scope of keeping

$$
\int_{\Omega}\left|\nabla\left(u-u_{h}\right)\right|^{p} \mathrm{~d} x
$$

infinitesimal, and letting

$$
\int_{S_{\varepsilon_{h} \delta}}\left|M_{n} \nabla u_{h}\right|^{\gamma} \mathrm{d} x
$$

diverge at a controlled rate, independently of the function $u$. Note that this is equivalent to show

$$
E_{h}\left(u_{h}, v_{h}, S_{\varepsilon_{h} \delta_{h}}\right)=\int_{S_{\varepsilon_{h} \delta_{h}}}\left|\nabla u_{h}\right|^{p}+k_{h}\left|M_{n} \nabla u_{h}\right|^{\gamma} \mathrm{d} x+W(1) \frac{\mathscr{L}^{m}\left(S_{\varepsilon_{h} \delta_{h}}\right)}{\varepsilon_{h}^{n}} \rightarrow 0
$$

for suitable $k_{h}$, because the last term is infinitesimal by (6.1). Suppose $\phi^{1}$ is a smooth function. If we multiply only the first coordinate by $\phi^{1}$ and compute the jacobian determinant we obtain

$$
\nabla\left(\phi^{1} u^{1}, u^{2}, \ldots, u^{n}\right)=\left(\phi^{1} \nabla u^{1}, \nabla u^{2}, \ldots, \nabla u^{n}\right)+\left(u^{1} \nabla \phi^{1}, \nabla u^{2}, \ldots, \nabla u^{n}\right),
$$

hence

$$
J\left(\phi^{1} u^{1}, u^{2}, \ldots, u^{n}\right)=\phi^{1} J u+u^{1} J\left(\phi^{1}, u^{2}, \ldots, u^{n}\right)
$$

in the sense of currents; also the following pointwise estimate holds for $1 \leq k \leq n$ :

$$
\begin{aligned}
& \left|M_{k} \nabla\left(\phi^{1} u^{1}, u^{2}, \ldots, u^{n}\right)\right| \\
& \leq\left.\left|\left(\begin{array}{c}
n-1 \\
k
\end{array}\right)\right| M_{k} \nabla u\right|^{2}+\left.\left(\begin{array}{c}
n-1 \\
k-1
\end{array}\right)\left(\left\|\phi^{1}\right\|_{L^{\infty}}\left|M_{k} \nabla u\right|+\left\|\nabla \phi^{1}\right\|_{L^{\infty}}\left|u^{1}\right|\left|M_{k-1} \nabla u\right|\right)^{2}\right|^{\frac{1}{2}} \\
& \quad \leq c_{n, k}\left(\left(1+\left\|\phi^{1}\right\|_{L^{\infty}}\right)\left|M_{k} \nabla u\right|+\left\|\nabla \phi^{1}\right\|_{L^{\infty}}\left|u^{1}\right|\left|M_{k-1} \nabla u\right|\right) .
\end{aligned}
$$


Therefore if we truncate $u$ by multiplying each component $u^{i}$ by smooth functions $\phi^{i}$ which satisfy $\operatorname{spt}\left(\nabla \phi^{i}\right) \cap$ $\operatorname{spt}\left(\nabla \phi^{j}\right)=\emptyset$ for $i \neq j$, we obtain that

$$
\begin{aligned}
& \phi \bowtie u:=\left(\phi^{1} u^{1}, \phi^{2} u^{2}, \ldots, \phi^{n} u^{n}\right)=0 \quad \text { in }\{\phi=0\}=\bigcap_{i}\left\{\phi^{i}=0\right\}, \\
& \left|M_{k} \nabla(\phi \bowtie u)\right| \leq c_{n, k}\left(\left(1+\|\phi\|_{L^{\infty}}\right)\left|M_{k} \nabla u\right|+\|\nabla \phi\|_{L^{\infty}}|u|\left|M_{k-1} \nabla u\right|\right)
\end{aligned}
$$

because at each point for only one index $j$ the gradient row $\nabla\left(\phi^{j} u^{j}\right)$ will present the non zero extra term $u^{j} \nabla \phi^{j}$. Observe also that (6.6) implies that

$$
\text { if } \quad S_{u} \Subset\{\phi=0\} \quad \text { then } J\left(\phi^{1} u^{1}, \ldots, \phi^{n} u^{n}\right) \ll \mathscr{L}^{m} .
$$

Finally note that if the supports of the gradients $\operatorname{spt}\left(\nabla \phi^{j}\right)$ overlap then the jacobian of $u \bowtie \phi$ will in general be bounded by the full vector of minors $M \nabla u$; however the particular choice where all $\phi^{i}$ 's are equal restores the dependence of the bound only on the precedent order minor, since the choice of $\nabla \phi$ in two rows annihilates the minor.

Choose functions $\phi_{h}=\left(\phi_{h}^{1}, \ldots, \phi_{h}^{n}\right)$ such that

- $0 \leq \phi_{h}^{i} \leq 1$;

- $\phi_{h}^{i}=1$ outside $S_{\left(2^{-1}+2^{-i}\right) \varepsilon_{h} \delta_{h}}$;

- $\phi_{h}^{i}=0$ inside $S_{\left(2^{-1}+2^{-i-1}\right) \varepsilon_{h} \delta_{h}}$;

- $\left|\nabla \phi_{h}^{i}\right| \leq 2^{i+2}\left(\varepsilon_{h} \delta_{h}\right)^{-1}$

and set $u_{h}:=\phi_{h} \bowtie u$. Then clearly $\left(u_{h}, v_{h}\right) \in Y(\Omega)$; note also that $u_{h} \rightarrow u$ in $L^{s}$ by dominated convergence. Moreover by the conditions on $\left(\phi_{h}^{i}\right)$, estimate (6.7) applied to $k=1$ (with the convention $M_{0} \nabla u=1$ ) reduces to $\left|\nabla u_{h}\right| \leq c_{n}\left(|\nabla u|+\left(\varepsilon_{h} \delta_{h}\right)^{-1}|u|\right)$ and yields

$$
\begin{aligned}
\int_{\Omega}\left|\nabla\left(u_{h}-u\right)\right|^{p} \mathrm{~d} x & \leq c_{n, p} \int_{S_{\varepsilon_{h} \delta_{h}}}|\nabla u|^{p} \mathrm{~d} x+c_{n, p}\left(\varepsilon_{h} \delta_{h}\right)^{-p} \int_{S_{\varepsilon_{h} \delta_{h}}}|u|^{p} \mathrm{~d} x \leq \\
& \leq c_{n, p} \int_{S_{\varepsilon_{h} \delta_{h}}}|\nabla u|^{p} \mathrm{~d} x+c_{n, p}\left(\varepsilon_{h} \delta_{h}\right)^{-p}\left(\int_{S_{\varepsilon_{h} \delta_{h}}}|u|^{\frac{n p}{n-p}} \mathrm{~d} x\right)^{\frac{n-p}{n}} \mathscr{L}^{m}\left(S_{\varepsilon_{h} \delta_{h}}\right)^{\frac{p}{n}} \\
& \leq c_{n, p} \int_{S_{\varepsilon_{h} \delta_{h}}}|\nabla u|^{p} \mathrm{~d} x+c_{n, p}\|u\|_{L^{\frac{n p}{n-p}}\left(S_{\varepsilon_{h} \delta_{h}}\right)}\left(1+\mathcal{M}^{* m-n}\left(S_{u}\right)\right)^{\frac{p}{n}}
\end{aligned}
$$

Therefore $u_{h}$ is close to $u$ in $W^{1, p}$. Regarding the jacobian term, we have:

Proposition 6.4. If $k_{h}\left(\varepsilon_{h} \delta_{h}\right)^{-\gamma} \rightarrow 0$ then

$$
\limsup _{h \rightarrow \infty} \int_{\Omega}\left(v_{h}+k_{h}\right)\left|M_{n} \nabla u_{h}\right|^{\gamma} \mathrm{d} x=\int_{\Omega}\left|M_{n} \nabla u\right|^{\gamma} \mathrm{d} x
$$

Proof. By construction $u_{h}=u$ outside $S_{\varepsilon_{h} \delta_{h}}$ and by Lebesgue dominated convergence Theorem

$$
\int_{\Omega \backslash S_{\varepsilon_{h} \delta_{h}}} v_{h}\left|M_{n} \nabla u_{h}\right|^{\gamma} \mathrm{d} x \rightarrow \int_{\Omega}\left|M_{n} \nabla u\right|^{\gamma} \mathrm{d} x .
$$

On the other hand inside $S_{\varepsilon_{h} \delta_{h}} v_{h}$ is identically zero, hence we are left with the estimate of $k_{h} \int_{S_{\varepsilon_{h} \delta_{h}}}\left|M_{n} \nabla u_{h}\right|^{\gamma} \mathrm{d} x$. Thanks to (6.7) we know that

$$
\int_{S_{\varepsilon_{h} \delta_{h}}}\left|M_{n} \nabla u_{h}\right|^{\gamma} \mathrm{d} x \leq c_{m, n, \gamma}\left(1+\left\|\phi_{h}\right\|_{L^{\infty}}\right)^{\gamma} \int_{S_{\varepsilon_{h} \delta_{h}}}\left|M_{n} \nabla u\right|^{\gamma} \mathrm{d} x+c_{m, n, \gamma}\left\|\nabla \phi_{h}\right\|_{L^{\infty}}^{\gamma} \int_{S_{\varepsilon_{h} \delta_{h}}}|u|^{\gamma}\left|M_{n-1} \nabla u\right|^{\gamma} \mathrm{d} x .
$$


The first term is infinitesimal by the absolute continuity of the integral. The second one can be estimated applying Hölder's inequality with exponents $\frac{s}{\gamma}$ and $\frac{p}{\gamma(n-1)}$ : this can be done because

$$
\frac{\gamma(n-1)}{p}+\frac{\gamma}{s} \leq 1
$$

Recalling Hadamard's inequality $\left|M_{k} \nabla u\right| \leq c_{k}|\nabla u|^{k}$ we get

$$
\int_{S_{\varepsilon_{h} \delta_{h}}}|u|^{\gamma}\left|M_{n-1} \nabla u\right|^{\gamma} \mathrm{d} x \leq c_{k}\|u\|_{L^{s}\left(S_{\varepsilon_{h} \delta_{h}}\right.}^{\gamma}\|\nabla u\|_{L^{p}\left(S_{\varepsilon_{h} \delta_{h}}\right)}^{\gamma(n-1)} .
$$

Since $\left\|\nabla \phi_{h}\right\|_{L^{\infty}}^{\gamma} \leq c\left(\varepsilon_{h} \delta_{h}\right)^{-\gamma}$ our assumption on $k_{h}$ allows to conclude.

Putting Propositions 6.2, 6.4 and (6.8) together we conclude the proof of Theorem 6.1.

Remark 6.5. From the proof of Theorem 3.7 we deduce that

$$
\liminf _{h} F_{h}\left(u_{h}, v_{h}, A\right) \geq F(u, A)
$$

for every open set $A \subset \Omega$, and

$$
\limsup _{h} F_{h}\left(u_{h}, v_{h}, \Omega\right) \leq F(u, \Omega) .
$$

This entails that $F_{h}\left(u_{h}, v_{h}, A\right) \rightarrow F(u, A)$ whenever $F(u, \partial A)=0$ and $\left(u_{h}, v_{h}\right) \rightarrow(u, 1)$ with equibounded energies. Note that $A \mapsto F(u, A)$ is the restriction to open sets of an absolutely continuous measure, hence it does not charge the boundary of any regular open set.

\subsection{Further observations}

It is interesting to notice that the exponent $\gamma$ is bounded above by $\frac{p}{n-1}$, in order for Theorem 6.1 to hold. There is however a trick allowing to overcome this bound, if we assume the Lagrangian to contain a nonlinear power of the full vector of minors $M \nabla u$ :

$$
|M \nabla u|=\left(\sum_{k=1}^{n}\left|M_{k} \nabla u\right|^{2}\right)^{\frac{1}{2}}
$$

Retaining the structure of the size and phase transition terms as in Definition 3.6, the bulk energy

$$
\tilde{F}(u, \Omega)=\int_{\Omega}|\nabla u|^{p}+|M \nabla u|^{\gamma} \mathrm{d} x
$$

can be approximated by

$$
\tilde{F}_{\varepsilon}(u, v, \Omega)=\int_{\Omega}|\nabla u|^{p}+\left.\left|\sum_{k=1}^{n-1}\right| M_{k} \nabla u\right|^{2}+\left.\left(v+k_{\varepsilon}\right)\left|M_{n} \nabla u\right|^{2}\right|^{\frac{\gamma}{2}} \mathrm{~d} x .
$$

Although $p>n-1$ guarantees that the same approximation holds, we can observe the following: applying Minkowski's inequality to (6.11) we have

$$
\begin{aligned}
k_{h}^{\frac{\gamma}{2}} \int_{S_{\varepsilon_{h} \delta_{h}}}\left|M_{n} \nabla u_{h}\right|^{\gamma} & \leq C_{\gamma} k_{h}^{\frac{\gamma}{2}} \int_{S_{\varepsilon_{\varepsilon} \delta_{h}}}\left|M_{n} \nabla u\right|^{\gamma}+\left|\nabla \phi_{h}\right|^{\gamma}|u|^{\gamma}\left|M_{n-1} \nabla u\right|^{\gamma} \mathrm{d} x \\
& \leq C_{\gamma}\left(1+k_{h}^{\frac{\gamma}{2}}\left\|\nabla \phi_{h}\right\|_{\infty}^{\gamma}\|u\|_{\infty}^{\gamma}\right) \tilde{F}\left(u, S_{\varepsilon_{h} \delta_{h}}\right) .
\end{aligned}
$$


Again if $k_{h}$ goes to 0 sufficiently fast then $k_{h}^{\frac{\gamma}{2}}\left\|\nabla \phi_{h}\right\|_{\infty}^{\gamma} \rightarrow 0$ and we get the $\Gamma$-upper limit statement, at least when $u \in L^{\infty}$. More generally the Lagrangian can feature different summability exponents on every order of the minors considered. In the model case

$$
\tilde{\tilde{F}}(u, \Omega):=\int_{\Omega}|\nabla u|^{p}+\sum_{k=2}^{n}\left|M_{k} \nabla u\right|^{p_{k}} \mathrm{~d} x
$$

Theorem 3.7 can be proved if we assume $p_{n}>1$ and (here $p_{1}=p$ )

$$
\frac{1}{s}+\frac{n-1}{p} \leq 1, \quad \frac{1}{s}+\frac{1}{p_{k-1}} \leq \frac{1}{p_{k}} .
$$

In particular if we impose $p<n$ to retain the possibility of $J u$ having a singular part, for the price of a very large $s$ we can take the $p_{k}$ 's arbitrarily close to the threshold $n$.

\section{BOUNDARY CONSTRAINTS}

In this section we analyse the behaviour of the previous $\Gamma$-convergence Theorems first when we compute the energy on subsets of the domain and then when we impose a boundary condition for $u$ at $\partial \Omega$ to be preserved by the approximating sequence. We start by applying the "free" version of the Theorem and combine it with Remark 3.2. If we want to prescribe a fixed trace at $\partial \Omega$ as observed in [10] the Sobolev trace constraint is not sufficient to properly set our problem, due to possible dependence of $J u$ on the exterior extension. We therefore set $U \ni \Omega$ open and fix $\phi \in W^{1, n}\left(U, \mathbb{R}^{n}\right)$ such that $\left.\phi\right|_{\partial \Omega} \in W^{1, p}\left(\partial \Omega, \mathbb{R}^{n}\right)$ : our approximating sequences will enjoy $u_{h}=\phi$ in $U \backslash \Omega$. Recall the previous result establishes the variational approximation of the energy on open sets: potential losses of mass due to presence of singular set at the boundary are disregarded in the lower limit, and a priori excluded in the upper limit by the hypothesis $\mathcal{M}_{\Omega}^{* m-n}\left(S_{u}\right)=\mathscr{H}^{m-n}\left(S_{u}\right)$.

The following proposition is an easy consequence of Theorem 3.7.

Proposition 7.1. Suppose $\left(\left(u_{h}, v_{h}\right)\right) \subset Y^{\phi}$ such that $\left(u_{h}, v_{h}\right) \rightarrow(u, v)$ and $\liminf _{h \rightarrow \infty} E_{h}\left(u_{h}, v_{h}, U\right)<\infty$. Then $v=1$ and

$$
\liminf _{h \rightarrow \infty} F_{h}\left(u_{h}, v_{h}, \Omega\right)+M M_{h}\left(v_{h}, U\right) \geq E(u, 1, \bar{\Omega})=F(u, 1, \Omega)+\sigma \mathscr{H}^{m-n}\left(S_{u} \cap \bar{\Omega}\right) .
$$

Proof. The statement follows straightforward from Theorem 3.7 applied to the domain $U$, since $S_{u} \subset \bar{\Omega} \Subset U$, hence $\mathscr{H}^{m-n}\left(S_{u} \cap U\right)=\mathscr{H}^{m-n}\left(S_{u} \cap \bar{\Omega}\right)$. Moreover Remark 6.5 entails that

$$
\liminf _{h} F_{h}\left(u_{h}, v_{h}, \Omega\right) \geq F(u, 1, \Omega),
$$

thus the proof is complete.

Similarly we can prove the upper limit analog:

Proposition 7.2. Suppose that $\Omega$ is of class $C^{2}, E(u, 1, U)<\infty$ and $\mathcal{M}^{* m-n}\left(S_{u}\right)=\mathscr{H}^{m-n}\left(S_{u}\right)$. Then there exists $\left(\left(u_{h}, v_{h}\right)\right) \subset Y^{\phi}$ such that

$$
\limsup _{h} E_{h}\left(u_{h}, v_{h}, \Omega\right) \leq E(u, 1, \bar{\Omega}) .
$$

Proof. Denote $\Omega_{s}=\{x \in U: \operatorname{sgndist}(x, \partial \Omega) \leq s\}$, where sgndist is the signed distance from $\partial \Omega$, positive outside $\Omega$ and negative inside. The $C^{2}$ regularity of $\Omega$ ensures the existence of a tubular neighborhood of $\partial \Omega$, namely there exists $s_{0}$ (depending on the $C^{2}$ norm of $\partial \Omega$ ) and a $C^{1}$ diffeomorphism

$$
\partial \Omega \times\left(-s_{0}, s_{0}\right) \ni(y, t) \mapsto x=y+t \nu(y) \in(\partial \Omega)_{s_{0}}
$$


built up via the normal map $\nu$ to $\partial \Omega$. With the help of this map one can construct, for any given $s \in\left(-s_{0}, s_{0}\right)$, Lipschitz diffeomorphisms $T_{s}: U \rightarrow U$ deforming $\Omega_{s}$ to $\Omega$ and satisfying $T_{0}=i d$ and

$$
\left\|T_{s}-T_{s^{\prime}}\right\|_{W^{1, \infty}(U, U)}+\left\|T_{s}^{-1}-T_{s^{\prime}}^{-1}\right\|_{W^{1, \infty}(U, U)} \leq C\left|s-s^{\prime}\right|
$$

for every $s, s^{\prime}$. We also point out that the existence of the tubular neighborhood gives a reflection map

$$
\Pi_{s_{0}}:(\partial \Omega)_{s_{0}} \ni(y, t) \mapsto(y,-t) \in(\partial \Omega)_{s_{0}}
$$

of class $C^{1}$ such that $\lim _{s_{0} \rightarrow 0}\left\|\Pi_{s_{0}}-i d\right\|_{C^{1}}=0$. Since the energy is finite $u \in G S B_{n} V(U)$ : given $\eta>0$ we let

$$
u_{\eta}= \begin{cases}u \circ T_{-\eta} & \text { in } \Omega_{-\eta}, \\ u \circ T_{s} & \text { on } \partial \Omega_{s},-\eta<s<0, \\ \phi & \text { in } U \backslash \Omega .\end{cases}
$$

Notice that $u_{\eta}=\phi$ outside $\Omega$ and $u_{\eta} \in W^{1, n}\left(U \backslash \Omega_{-\eta}, \mathbb{R}^{n}\right)$, hence $u_{\eta} \in X^{\phi}$ and $S_{u_{\eta}} \subset \overline{\Omega_{-\eta}} \Subset \Omega$. Moreover it is not difficult to use (7.2) to show $E\left(u_{\eta}, 1, U\right) \rightarrow E(u, 1, U)$ for $\eta \downarrow 0$ : in fact the energy in $U \backslash \Omega$ is fixed, the one in $\overline{\Omega_{-\eta}}$ after a change of variables equals to

$$
\begin{aligned}
\int_{\Omega}\left\{\left|\nabla u \cdot\left(D T_{-\eta} \circ T_{-\eta}^{-1}\right)\right|^{p}\right. & \left.+\left.\left.\left|\sum_{|I|=|J|=n}\right| \sum_{|K|=n} \operatorname{det}(\nabla u)_{K}^{I} \operatorname{det}\left(D T_{-\eta} \circ T_{-\eta}^{-1}\right)_{J}^{K}\right|^{2}\right|^{\frac{\gamma}{2}}\right\}\left|\operatorname{det} D T_{-\eta}^{-1}\right| \mathrm{d} x \\
& +\int_{\bar{\Omega} \cap S_{u}}\left|\left\langle\Lambda_{m-n} D T_{-\eta}^{-1}, \tau_{S_{u}}\right\rangle\right| \mathrm{d} \mathscr{H}^{m-n}
\end{aligned}
$$

which is asymptotically equal to $E(u, \bar{\Omega})$ thanks to (7.2); finally in the annulus $\Omega \backslash \overline{\Omega_{-\eta}}, u_{\eta}$ being a constant extension along the trajectories $s \mapsto T_{s}(x)$, enjoys

$$
\int_{\Omega \backslash \overline{\Omega_{-\eta}}}\left|\nabla u_{\eta}\right|^{p} \mathrm{~d} x \leq C(\partial \Omega) \eta \int_{\partial \Omega}\left|\nabla_{\tau} \phi\right|^{p} \mathrm{~d} x
$$

and $M_{n} \nabla u_{\eta}=0$, hence $E\left(u, 1, \Omega \backslash \overline{\Omega_{-\eta}}\right) \rightarrow 0$. Thanks to Remark 3.2 and Proposition 7.1 it is sufficient to prove the $\Gamma-\limsup$ for $u_{\eta}$. Theorem 6.1 ensures the existence of $\left(u_{h}, v_{h}\right) \in Y^{\phi}$ satisfying

$$
\underset{h}{\limsup } E_{h}\left(u_{h}, v_{h}, U\right) \leq E(u, 1, U):
$$

subtracting the constant term $F(\phi, 1, U \backslash \Omega)$ we have the thesis.

Propositions 7.1 and 7.2 are only in part satisfactory, since in (7.1) we took into account some energy outside $\Omega$. We want to refine these results assessing the quantitative loss of energy due to exterior phase transition in $M M_{h}$.

Proposition 7.3. With the same hypotheses of Proposition 7.1 it holds:

$$
\liminf _{h} M M_{h}\left(v_{h}, \Omega\right) \geq \sigma \mathscr{H}^{m-n}\left(S_{u} \cap \Omega\right)+\frac{1}{2} \sigma \mathscr{H}^{m-n}\left(S_{u} \cap \partial \Omega\right) .
$$

Proof. Let us start from the codimension zero case $m=n$. The proof stems from Lemma 5.5, applied to the larger domain $U$, whose argument we here retrace. Since we are evaluating the energy $M M_{h}\left(v_{h}, \Omega \cap B_{2 \rho}\left(x_{0}\right)\right)$ we can suppose $x_{0}=0 \in S_{u} \cap \partial \Omega$, as the interior case is already contained in Lemma 5.5. Recall the proof showed that in every ball $B_{\rho}(0) \subset U$ the sequence satisfies $\lim _{h} \inf _{B_{\rho}} v_{h}=0$. We actually know that

$$
\lim _{h} \inf _{B_{\rho} \cap \bar{\Omega}} v_{h}=0
$$


because every $u_{h}$ equals $\phi$ in $U \backslash \Omega$ and $J \phi \ll \mathscr{L}^{n}$. Let $\left(x_{h}\right)$ be one of the minimum points of $v_{h}$ in $B_{\rho}$ : we have two cases.

Case 1. $\left(\liminf \operatorname{ix}_{h} \frac{\left|x_{h}\right|}{\varepsilon_{h}}\right)<\infty$. In this case scaling back $v_{h}$ by a factor $\varepsilon_{h}$ we obtain

$$
M M_{h}\left(v_{h}, \Omega \cap B_{2 \rho}\right)=M M_{1}\left(v_{h}\left(\varepsilon_{h} x\right), \frac{\Omega \cap B_{2 \rho}}{\varepsilon_{h}}\right) .
$$

Using a diagonal argument and reasoning as in Proposition 4.1 we produce a limit $f_{\infty}$ such that

$$
v_{h}\left(\varepsilon_{h} x\right) \rightarrow 1-f_{\infty}(x) \quad \text { locally uniformly in } \mathbb{R}^{n}
$$

and

$$
\min _{\mathbb{R}^{n}}\left\{1-f_{\infty}\right\}=0
$$

Fix a compact $K \Subset H:=\{\langle x, \nu(0)\rangle<0\}$ : by $C^{1}$ regularity $\frac{\Omega}{\varepsilon_{h}} \rightarrow H$ locally in the Hausdorff metric and $K \subset \frac{1}{\varepsilon_{h}}\left(\Omega \cap B_{2 \rho}\right)$ for $h$ large enough. By lower semicontinuity

$$
\liminf _{h} M M_{1}\left(v_{h}\left(\varepsilon_{h} x\right), \frac{\Omega \cap B_{2 \rho}}{\varepsilon_{h}}\right) \geq \liminf _{h} M M_{1}\left(v_{h}\left(\varepsilon_{h} x\right), K\right) \geq M M_{1}\left(1-f_{\infty}, K\right)=I\left(f_{\infty}, K\right)
$$

and letting $K \uparrow H$ we entail

$$
\liminf _{h} M M_{h}\left(v_{h}, \Omega \cap B_{2 \rho}\right) \geq I\left(f_{\infty}, H\right) .
$$

Therefore if we redefine $f_{\infty}$ in $\mathbb{R}^{n} \backslash H$ by reflection with respect to $\partial H$ we obtain $I\left(f_{\infty}, H\right)=\frac{1}{2} I\left(f_{\infty}, \mathbb{R}^{n}\right)$. A radial rearrangement $f_{\infty}^{*}$ of $f_{\infty}$ decreases the energy and gives $f_{\infty}^{*}(0)=1$, hence by Proposition $4.1 I\left(f_{\infty}, H\right) \geq \frac{1}{2} \sigma$.

Case 2. $\lim _{h} \frac{\left|x_{h}\right|}{\varepsilon_{h}}=\infty$. In this situation we blow-up around $x_{h}$ and obtain that

$$
\frac{\Omega \cap B_{2 \rho}(0)-x_{h}}{\varepsilon_{h}} \supset B_{\frac{\rho}{\varepsilon_{h}}}(0) \rightarrow \mathbb{R}^{n}
$$

in the same sense as before. The limit $f_{\infty}$ of the translated sequence $\left(v_{h}\left(x_{h}+\varepsilon_{h} y\right)\right)$ will now satisfy $f_{\infty}(0)=0$, hence by lower semicontinuity

$$
\liminf _{h} M M_{1}\left(v_{h}\left(x_{h}+\varepsilon_{h} y\right), \frac{\Omega \cap B_{2 \rho}(0)-x_{h}}{\varepsilon_{h}}\right) \geq I\left(f_{\infty}, \mathbb{R}^{n}\right) \geq \sigma .
$$

The case $m>n$ can be treated as in (5.12), where now the projection measures $\tau_{\pi}$ contain the extra term $\frac{1}{2} \sigma \mathscr{H}^{m-n}\left\llcorner\left(S_{u} \cap \partial \Omega\right)\right.$.

Similarly we have a statement for the upper limit:

Proposition 7.4. For every $u \in X^{\phi}$ such that $E(u, 1, \Omega)<\infty, \mathcal{M}^{*}\left(S_{u}\right)=\mathscr{H}^{m-n}\left(S_{u}\right)$ and $\mathscr{H}^{m-n}\left(\overline{S_{u} \cap \Omega} \cap\right.$ $\partial \Omega)=0$ there exists a sequence $\left(\left(u_{h}, v_{h}\right)\right) \subset Y^{\phi}$ such that $\left(u_{h}, v_{h}\right) \rightarrow(u, 1)$ and

$$
\limsup _{h \rightarrow \infty} E_{h}\left(u_{h}, v_{h}, \Omega\right) \leq E(u, 1, \Omega)+\frac{1}{2} \sigma \mathscr{H}^{m-n}\left(S_{u} \cap \partial \Omega\right) .
$$

In order to prove this result we begin with a Lemma:

Lemma 7.5. Let $\tau>0$ be a given positive number: there exists a profile $\bar{w}:[0, \infty) \rightarrow[0,1]$ such that

1. $|I(\bar{w}(|x|))-\sigma|<\tau$;

2. $\bar{Z}(t):=\left|\bar{w}^{\prime}(t)\right|^{q}+W(\bar{w}(t))$ is decreasing;

3. $\bar{w} \in \operatorname{Lip}([0, \infty))$ and $\bar{w}=0$ in $[R, \infty)$ for some $R$; 
Proof. Using the optimal profile $w$ given by Proposition 4.1, it is sufficient to take into account the continuity of $I$ along the family of profiles

$$
\frac{w(t+\lambda)}{w(\lambda)}, \quad \lambda \geq 0
$$

and choose a $\lambda>0$ satisfying $\left|I\left(\frac{w(|x|+\lambda)}{w(\lambda)}\right)-\sigma\right|<\tau$. We name $\bar{w}$ the profile (7.5) relative to such choice: $\bar{w}$ is clearly Lipschitz by Lemma 4.2. The second property follows from the fact that both $w(t)$ and $\left|w^{\prime}(t)\right|$ are decreasing. The third one can be obtained again by dilating the new profile around 1 and truncate it to 0 changing the energy $I$ only of a small amount.

We will also use the following fact, whose proof we leave to the reader:

Lemma 7.6. If $S \subset \Omega$ is countably $\mathscr{H}^{k}$-rectifiable and satisfies $\mathcal{M}_{\Omega}^{* k}(S)=\mathscr{H}^{k}(S)$ then the same is true for every $S^{\prime} \subset S$ such that $\mathscr{H}^{k}\left(S \cap\left(\overline{S^{\prime}} \backslash S^{\prime}\right)\right)=0$.

We can now prove Proposition 7.4.

Proof. By the finiteness of the energy $u \in G S B_{n} V(U)$ and $S_{u} \subset \bar{\Omega}$. Let $\eta_{h} \downarrow 0$ to be chosen later. We can consider the tilted sequence $u_{\eta_{h}}$ described in (7.3): we have

$$
\lim _{h} F\left(u_{\eta_{h}}, 1, \Omega\right)=F(u, 1, \Omega) .
$$

For an arbitrary $\tau$ let $\bar{w}$ be a function as in Lemma 7.5: by Proposition 6.2 and Remark 6.3 we can construct a sequence $\left(v_{h}\right)$ of approximating functions such that

$$
\underset{h}{\limsup } M M_{h}\left(v_{h}, \Omega\right)=I(\bar{w}(|x|)) \mathcal{M}_{\Omega}^{* m-n}\left(S_{u}\right)<(\sigma+\tau) \mathcal{M}_{\Omega}^{* m-n}\left(S_{u}\right) .
$$

Denote $v_{\eta_{h}, h}=v_{h} \circ T_{-\eta_{h}}$ : recall that $v_{h}=0$ in $S_{\varepsilon_{h} \delta_{h}}$ and because of (7.2) we have that

$$
\| T_{-\eta_{h}}^{-1}(x)-T_{-\eta_{h}}^{-1}(y)|-| x-y|| \leq \operatorname{Lip}\left(T_{-\eta_{h}}^{-1}-i d\right)|x-y| \leq C \eta_{h}|x-y|,
$$

therefore $v_{\eta_{h}, h}=0$ on $\left(T_{-\eta_{h}}^{-1}\left(S_{u}\right)\right)_{\varepsilon_{h} \delta_{h}\left(1-C \eta_{h}\right)}$, thus eventually in $\left(T_{-\eta_{h}}^{-1}\left(S_{u}\right)\right)_{\varepsilon_{h} \delta_{h} / 2}$.

Let us analyse the bulk part first. Since the null set of $v_{\eta_{h}, h}$ has width at least $\varepsilon_{h} \delta_{h} / 2$ we can apply Theorem 6.1 relative to the limit $u_{\eta_{h}}$ in the domain $U$ and define $u_{\eta_{h}, h}$ such that

- $\left(u_{\eta_{h}, h}, v_{\eta_{h}, h}\right) \in Y(U)$,

- $\left|u_{\eta_{h}, h}\right| \leq\left|u_{\eta_{h}}\right|$ pointwise almost everywhere,

- $u_{\eta_{h}, h}=u_{\eta_{h}}$ outside $\left(T_{-\eta_{h}}^{-1}\left(S_{u}\right)\right)_{\varepsilon_{h} \delta_{h} / 2} \subset\left\{v_{\eta_{h}, h}=0\right\}$.

In particular $u_{\eta_{h}, h} \rightarrow u$ in $L^{s}$. Moreover the construction guarantees that

$$
\begin{aligned}
\left.\left|F_{h}\left(u_{\eta_{h}, h}, v_{h}, U\right)-\int_{U}\right| \nabla u_{\eta_{h}}\right|^{p}+v_{h}\left|M_{n} \nabla u_{\eta_{h}}\right|^{\gamma} \mathrm{d} x \mid & \\
& \leq \int_{U} k_{h}\left|M_{n} \nabla u_{\eta_{h}, h}\right|^{\gamma} \mathrm{d} x+\int_{\left(T_{-\eta_{h}}^{-1}\left(S_{u}\right)\right)_{\varepsilon_{h} \delta_{h} / 2}}\left|\nabla u_{\eta_{h}, h}\right|^{p}+\left|\nabla u_{\eta_{h}}\right|^{p} \mathrm{~d} x
\end{aligned}
$$

The same estimates yielding (6.8) and (6.9) show that the right hand side is infinitesimal. Furthermore the constraint $\left(u_{\eta_{h}, h}, v_{\eta_{h}, h}\right) \in Y^{\phi}$ is satisfied once we choose $\eta_{h}=\varepsilon_{h} \delta_{h}$. Observing that

$$
\int_{U}\left|\nabla u_{\eta_{h}}\right|^{p}+v_{h}\left|M_{n} \nabla u_{\eta_{h}}\right|^{\gamma} \mathrm{d} x \leq F\left(u_{\eta_{h}}, 1, U\right) \rightarrow F(u, 1, U)
$$


the previous two equations entail

$$
\limsup F_{h}\left(u_{\eta_{h}, h}, v_{h}, U\right) \leq F(u, 1, U) .
$$

Subtracting the constant term $F(u, 1, U \backslash \Omega)$ we remain with

$$
\begin{aligned}
F(u, 1, \Omega) & \geq \limsup _{h} F_{h}\left(u_{\eta_{h}, h}, v_{h}, U\right)-F(u, 1, U \backslash \Omega) \\
& =\limsup _{h} F_{h}\left(u_{\eta_{h}, h}, v_{h}, \Omega\right)+F_{h}\left(u_{\eta_{h}, h}, v_{h}, U \backslash \Omega\right)-F(u, 1, U \backslash \Omega) \\
& =\limsup _{h} F_{h}\left(u_{\eta_{h}, h}, v_{h}, \Omega\right)+F_{h}\left(u, v_{h}, U \backslash \Omega\right)-F(u, 1, U \backslash \Omega) \\
& =\limsup _{h} F_{h}\left(u_{\eta_{h}, h}, v_{h}, \Omega\right)+\int_{U \backslash \Omega}\left(v_{h}+k_{h}-1\right)\left|M_{n} \nabla u\right|^{\gamma} \mathrm{d} x \\
& =\limsup _{h} F_{h}\left(u_{\eta_{h}, h}, v_{h}, \Omega\right) .
\end{aligned}
$$

It remains to evaluate the asymptotic of $M M_{h}\left(v_{\eta_{h}, h}, \Omega\right)$. First of all changing back variables we have that

$$
M M_{h}\left(v_{\eta_{h}, h}, \Omega\right)=\int_{T_{\eta_{h}}(\Omega)}\left\{\varepsilon_{h}^{q-n}\left|\left(D T_{-\eta_{h}} \circ T_{-\eta_{h}}^{-1}\right) \nabla v_{h}\right|^{q}+\frac{W\left(1-v_{h}\right)}{\varepsilon_{h}^{n}} \mathrm{~d} x\right\}\left|\operatorname{det} D T_{-\eta_{h}}^{-1}\right| \mathrm{d} x
$$

and by (7.2) and Lemma 7.5 this is asymptotic to $M M_{h}\left(v_{h}, T_{\eta_{h}}(\Omega)\right)$ : we now show that if $\frac{\eta_{h}}{\varepsilon_{h}}=\delta_{h} \rightarrow 0$ sufficiently fast then the last energy is asymptotically equal to $M M_{h}\left(v_{h}, \Omega\right)$, namely $M M_{h}\left(v_{h}, T_{\eta_{h}}(\Omega) \backslash \Omega\right) \rightarrow 0$.

Fix a radius $R$ such that $\operatorname{spt}(\bar{w}) \subset B_{R}^{n}$ and $\mathscr{L}^{m}\left(B_{2 \varepsilon_{h} R} \cap\left(T_{\eta_{h}}(\Omega) \backslash \Omega\right)\right) \leq C\left(\varepsilon_{h} R\right)^{m-1} \eta_{h}$. We can cover $S_{\varepsilon_{h} R}$ with (closed) balls of radius $\varepsilon_{h} R$ centered at $x_{0} \in S_{u}$ :

$$
S_{\varepsilon_{h} R} \subset \bigcup_{x_{0} \in S_{u}} \overline{B_{\varepsilon_{h} R}\left(x_{0}\right)} .
$$

By Besicovitch's covering Lemma there are $N$ disjoint subfamilies $\mathcal{F}_{i}$ that still cover the set of old centers, namely $S_{u}$ : by triangle inequality

$$
S_{\varepsilon_{h} R} \subset \bigcup_{i=1}^{N} \bigcup_{\mathcal{F}_{i}} B_{2 \varepsilon_{h} R}
$$

and the assumption of $\mathcal{M}^{* m-n}\left(S_{u}\right)$ implies that $\# \mathcal{F}_{i} \leq C\left(\varepsilon_{h} R\right)^{n-m}$; as a consequence the family of double balls $\left\{B_{2 \varepsilon_{h} R}\right\}$ has bounded overlap. Without loss of generality we can also assume that $\mathcal{M}^{* m-n}\left(S_{u} \cap B_{2 \varepsilon_{h} R}\left(x_{0}\right)\right)=$ $\mathscr{H}^{m-n}\left(S_{u} \cap B_{2 \varepsilon_{h} R}\left(x_{0}\right)\right)$, recalling that this is true at almost every radius. For any of such double ball

$$
\frac{M M_{h}\left(v_{h}, B_{2 \varepsilon_{h} R}\left(x_{0}\right) \cap\left(T_{\eta_{h}}(\Omega) \backslash \Omega\right)\right)}{\varepsilon_{h}^{m-n}}=\int_{B_{2 R}(0) \cap \frac{\left(T_{\eta_{h}}(\Omega) \backslash \Omega\right)-x_{0}}{\varepsilon_{h}}}\left|\nabla \psi_{h}\right|^{q}+W\left(\psi_{h}\right) \mathrm{d} y
$$

with

$$
\psi_{h}(y)=\bar{w}_{h}\left(\mathrm{~d}\left(y, \frac{S_{u}-x_{0}}{\varepsilon_{h}}\right)\right) .
$$

The integral can be simply bounded by

$$
\left(\operatorname{Lip}\left(\bar{w}_{h}\right)^{q}+\|W\|_{\infty}\right) \mathscr{L}^{m}\left(B_{2 R}(0) \cap \frac{\left(T_{\eta_{h}}(\Omega) \backslash \Omega\right)-x_{0}}{\varepsilon_{h}}\right) \leq C\left(\operatorname{Lip}\left(\bar{w}_{h}\right)^{q}+\|W\|_{\infty}\right) R^{m-1} \frac{\eta_{h}}{\varepsilon_{h}} .
$$

Recall the construction of $\bar{w}_{h}$ from $\bar{w}$ in $(6.3)$ gives that $\operatorname{Lip}\left(\bar{w}_{h}\right) \leq C \operatorname{Lip}(\bar{w})$. Summing on the number of balls we have

$$
M M_{h}\left(v_{h}, \Omega_{\eta_{h}} \backslash \Omega\right) \leq C \varepsilon_{h}^{m-n}\left(\varepsilon_{h} R\right)^{n-m}\left(\operatorname{Lip}\left(\bar{w}_{h}\right)^{q}+\|W\|_{\infty}\right) R^{m-1} \frac{\eta_{h}}{\varepsilon_{h}}=C\left(\operatorname{Lip}(\bar{w})^{q}+\|W\|_{\infty}\right) R^{n-1} \frac{\eta_{h}}{\varepsilon_{h}} \rightarrow 0 .
$$


Suppose now that $\mathscr{H}^{m-n}\left(\overline{S_{u} \cap \Omega} \cap \partial \Omega\right)=0$ : then $\mathcal{M}_{U}^{* m-n}\left(S_{u} \cap \Omega\right)=\mathscr{H}^{m-n}\left(S_{u} \cap \Omega\right)$ by Lemma 7.6 (applied to $\left.S^{\prime}=S_{u} \cap \Omega\right)$. Moreover:

$$
\mathcal{M}_{\Omega}^{* m-n}\left(S_{u}\right) \leq \mathcal{M}_{U}^{* m-n}\left(S_{u} \cap \Omega\right)+\mathcal{M}_{\Omega}^{* m-n}\left(S_{u} \cap \partial \Omega\right)=\mathscr{H}^{m-n}\left(S_{u} \cap \Omega\right)+\mathcal{M}_{\Omega}^{* m-n}\left(S_{u} \cap \partial \Omega\right) .
$$

Regarding the last term, by (7.2) the reflection map $\Pi_{s_{0}}$ that swaps $\Omega$ and $U \backslash \Omega$ has a jacobian uniformly close to 1 as we move close to $\partial \Omega$ and therefore

$$
\mathcal{M}_{\Omega}^{* m-n}\left(S_{u} \cap \partial \Omega\right)=\frac{1}{2} \mathcal{M}_{U}^{* m-n}\left(S_{u} \cap \partial \Omega\right)=\frac{1}{2} \mathscr{H}^{m-n}\left(S_{u} \cap \partial \Omega\right) .
$$

In conclusion

$$
\limsup _{h} M M_{h}\left(v_{h}, \Omega\right) \leq(\sigma+\tau)\left(\mathscr{H}^{m-n}\left(S_{u} \cap \Omega\right)+\frac{1}{2} \mathscr{H}^{m-n}\left(S_{u} \cap \partial \Omega\right)\right)
$$

and the assertion follows by letting $\tau \rightarrow 0$.

\section{General Lagrangians}

The $\Gamma$-convergence Theorem 3.7 proved in the previous sections can be extended, always in the setting of higher codimension singular sets, to polyconvex Lagrangians of more general form than Definition 2.10.

Indeed the key ingredients for the $\Gamma$ - lim inf are again the compactness Theorem 2.8 , which is at the heart of Theorem 2.11, as well as the lower semicontinuity of the energy for the convergence provided by it. Regarding the $\Gamma$-limsup in order to approximate the size term we rely on the same Modica-Mortola approximation of before. The recovery sequence is obtained via an approximation in measure of the limit function $u$, with regular functions $u_{\varepsilon} \in R_{n}$ coinciding with $u$ outside the narrow sets $S_{\varepsilon}$. The proof of Proposition 6.4 amounts to show that the contribution to the bulk energy in $S_{\varepsilon}$ is infinitesimal.

Both these arguments can be adapted to a broader class of Lagrangians that we now present (see [10] for the study of the relative Dirichlet and Neumann problems). We denote $\mathscr{L}_{m}$ the $\sigma$-algebra of Lebesgue measurable subsets of $\mathbb{R}^{m}$ and $\mathscr{B}\left(\mathbb{R}^{n+\kappa}\right)$ the $\sigma$-algebra of Borel subsets of $\mathbb{R}^{n+\kappa}$. Assume the following hypotheses on the functions $f: \Omega \times \mathbb{R}^{n} \times \mathbb{R}^{\kappa} \rightarrow[0,+\infty)$ and $g: \Omega \rightarrow[0, \infty)$ are satisfied:

(a) $f$ is $\mathscr{L}_{m} \times \mathscr{B}\left(\mathbb{R}^{n+\kappa}\right)$-measurable;

(b) for $\mathscr{L}^{m}$-a.e. $x \in \Omega,(u, w) \mapsto f(x, u, w)$ is lower semicontinuous;

(c) for $\mathscr{L}^{m}$-a.e. $x \in \Omega$ and for every $u \in \mathbb{R}^{n}$ the map $w \mapsto f(x, u, w)$ is convex in $\mathbb{R}^{\kappa}$;

(d) $c\left(\left|w_{1}\right|^{p}+\Psi\left(\left|w_{n}\right|\right)\right) \leq f(x, u, w) \leq C\left(1+|u|^{s}+\left|w_{1}\right|^{p}+\left|w_{n}\right|^{\gamma}\right)$ for $\Psi$ convex and superlinear at infinity and for some constants $\gamma>1, c, C>0$;

and $g \in C^{0}(\bar{\Omega}), g \geq c>0$. Our energy is:

$$
\mathcal{E}(u, \Omega)=\int_{\Omega} f(x, u, M \nabla u) \mathrm{d} x+\sigma \int_{\Omega \cap S_{u}} g \mathrm{~d} \mathscr{H}^{m-n} .
$$

Thanks to the Theorem 2.8 the energy (8.1) is lower semicontinuous along sequences converging strongly in $L^{s}$ and with equibounded energies. The upper bound on $f$ on the other side allows to prove the upper limit statement. The approximating energies will be

$$
\mathcal{E}_{\varepsilon}(u, v, \Omega):=\int_{\Omega} f\left(x, u, \nabla u, \ldots, M_{n-1} \nabla u,\left(v+k_{\varepsilon}\right) M_{n} \nabla u\right) \mathrm{d} x+\int_{\Omega} g(x)\left(\varepsilon^{q-n}|\nabla v|^{q}+\frac{W(1-v)}{\varepsilon^{n}}\right) \mathrm{d} x .
$$

We therefore have:

Theorem 8.1. Let $\Omega$ be a bounded open subset of class $C^{1}$ of $\mathbb{R}^{m}$ and suppose

$$
s \geq \frac{n p}{n-p}, \quad 1<\gamma \leq \frac{1}{\frac{n-1}{p}+\frac{1}{s}}, \quad q>n, \quad k_{\varepsilon}=o(\varepsilon) .
$$


Suppose the integrands $f, g$ satisfy the assumptions above. Then:

(a) For every sequence $\left(\left(u_{h}, v_{h}\right)\right) \subset Y(\Omega)$ such that $\liminf _{h \rightarrow \infty} \mathcal{E}_{h}\left(u_{h}, v_{h}, \Omega\right)<\infty$ and $\left(u_{h}, v_{h}\right) \rightarrow(u, v)$ in $X(\Omega)$ we have

$$
u \in G S B_{n} V(\Omega), v=1 \text { and } \liminf _{h \rightarrow \infty} \mathcal{E}_{h}\left(u_{h}, v_{h}, \Omega\right) \geq \mathcal{E}(u, \Omega) .
$$

(b) For every $u \in G S B_{n} V(\Omega)$ such that $\mathcal{E}(u, 1, \Omega)<\infty$ and $\mathcal{M}_{\Omega}^{* m-n}\left(S_{u}\right)=\mathscr{H}^{m-n}\left(S_{u}\right)$ there exists a sequence $\left(\left(u_{h}, v_{h}\right)\right) \subset Y(\Omega)$ such that $\left(u_{h}, v_{h}\right) \rightarrow(u, 1)$ in $X(\Omega)$ and

$$
\limsup _{h \rightarrow \infty} \mathcal{E}_{h}\left(u_{h}, v_{h}, \Omega\right) \leq \mathcal{E}(u, \Omega)
$$

Acknowledgements. The author wishes to thank his advisor prof.Luigi Ambrosio for encouraging him through the development of this project; he is also grateful to Giovanni Alberti, Camillo De Lellis, Guido De Philippis, Nicola Fusco, Bernd Kirchheim, Emanuele Spadaro and Bozhidar Velichkov for many useful discussions. The author acknowledges the support of ERC ADG GeMeThNES.

\section{REFERENCES}

[1] E. Acerbi and G. Dal Maso, New lower semicontinuity results for polyconvex integrals. Calc. Var. Partial Differ. Equ. 2 (1994) 329-371.

[2] R.A. Adams and J.J.F. Fournier, Sobolev spaces, Pure and Applied Mathematics (Amsterdam), vol. 140, 2nd edition. Elsevier/Academic Press, Amsterdam (2003).

[3] G. Alberti, S. Baldo and G. Orlandi, Functions with prescribed singularities. J. Eur. Math. Soc. (JEMS) 5 (2003) $275-311$.

[4] G. Alberti, S. Baldo and G. Orlandi, Variational convergence for functionals of Ginzburg-Landau type. Indiana Univ. Math. J. 54 (2005) 1411-1472.

[5] F. Almgren. Deformations and multiple-valued functions, in Geometric measure theory and the calculus of variations (Arcata, Calif., 1984), vol. 44 of Proc. Sympos. Pure Math. Amer. Math. Soc. Providence, RI (1986) 29-130.

[6] L. Ambrosio, Existence theory for a new class of variational problems. Arch. Rational Mech. Anal. 111 (1990) $291-322$.

[7] L. Ambrosio, Metric space valued functions of bounded variation. Ann. Scuola Norm. Sup. Pisa Cl. Sci. 17 (1990) 439-478.

[8] L. Ambrosio, N. Fusco and D. Pallara, Functions of bounded variation and free discontinuity problems, Oxford Mathematical Monographs. The Clarendon Press Oxford University Press, New York (2000).

[9] L. Ambrosio and F. Ghiraldin, Flat chains of finite size in metric spaces. Annales de l'Institut Henri Poincaré (C) Non Linear Analysis (2012).

[10] L. Ambrosio and F. Ghiraldin, Compactness of special functions of bounded higher variation. Analysis and Geometry in Metric Spaces 1 (2013) 1-30.

[11] L. Ambrosio and B. Kirchheim, Currents in metric spaces. Acta Math. 185 (2000) 1-80.

[12] L. Ambrosio and V.M. Tortorelli, Approximation of functionals depending on jumps by elliptic functionals via $\Gamma$-convergence. Comm. Pure Appl. Math. 43 (1990) 999-1036.

[13] L. Ambrosio and V.M. Tortorelli, On the approximation of free discontinuity problems. Boll. Un. Mat. Ital. B 6 (1992) $105-123$.

[14] J.M. Ball, Convexity conditions and existence theorems in nonlinear elasticity. Arch. Rational Mech. Anal. 63 (1976/1977) $337-403$.

[15] F. Bethuel, H. Brezis and F. Hélein, Ginzburg-Landau vortices, vol. 13 of Progress in Nonlinear Differential Equations and their Applications. Birkhäuser Boston Inc., Boston, MA (1994).

[16] A. Braides, $\Gamma$-convergence for beginners, vol. 22 of Oxford Lecture Series in Mathematics and its Applications. Oxford University Press, Oxford (2002).

[17] G. Dal Maso, An introduction to $\Gamma$-convergence. Progr. Nonlinear Differ. Eq. Appl., vol. 8. Birkhäuser Boston Inc., Boston, MA (1993).

[18] G. David, Singular sets of minimizers for the Mumford-Shah functional, vol. 233 of Progress in Mathematics. Birkhäuser Verlag, Basel (2005).

[19] E. De Giorgi, M. Carriero and A. Leaci, Existence theorem for a minimum problem with free discontinuity set. Arch. Rational Mech. Anal. 108 (1989) 195-218.

[20] C. De Lellis, Some fine properties of currents and applications to distributional Jacobians. Proc. Roy. Soc. Edinburgh Sect. A 132 (2002) 815-842.

[21] C. De Lellis and F. Ghiraldin, An extension of the identity Det = det. C. R. Acad. Sci. Paris Sér. I Math. (2010).

[22] T. De Pauw and R. Hardt. Rectifiable and flat $G$ chains in a metric space. Amer. J. Math. 134 (2012) 1-69.

[23] N. Desenzani and I. Fragalà, Concentration of Ginzburg-Landau energies with supercritical growth. SIAM J. Math. Anal. 38 (2006) 385-413 (electronic). 
[24] H. Federer, Geometric measure theory, vol. 153 of Die Grundlehren der mathematischen Wissenschaften, Band. SpringerVerlag New York Inc., New York (1969).

[25] H. Federer, Flat chains with positive densities. Indiana Univ. Math. J. 35 (1986) 413-424.

[26] W. H. Fleming, Flat chains over a finite coefficient group. Trans. Amer. Math. Soc. 121 (1966) 160-186.

[27] N. Fusco and J.E. Hutchinson, A direct proof for lower semicontinuity of polyconvex functionals. Manuscripta Math. 87 (1995) $35-50$.

[28] M. Giaquinta, G. Modica and J. Souček, Cartesian currents in the calculus of variations. I, II, vol. 37, 38 of Ergebnisse der Mathematik und ihrer Grenzgebiete 3. Folge. A Series of Modern Surveys in Mathematics [Results in Mathematics and Related Areas. 3rd Series. A Series of Modern Surveys in Mathematics]. Springer-Verlag, Berlin (1998).

[29] Enrico Giusti, Direct methods in the calculus of variations. World Scientific Publishing Co. Inc., River Edge, NJ (2003). MR 1962933 (2004g:49003)

[30] R. Hardt and T. Rivière, Connecting topological Hopf singularities. Ann. Sc. Norm. Super. Pisa Cl. Sci. 2 (2003) $287-344$.

[31] R.L. Jerrard and H.M. Soner, Functions of bounded higher variation. Indiana Univ. Math. J. 51 (2002) 645-677.

[32] E.H. Lieb and M. Loss, Analysis, Graduate Studies in Mathematics, vol. 14 of Amer. Math. Soc. Providence, RI, 2nd edition (2001).

[33] L. Modica and S. Mortola, Il limite nella $\Gamma$-convergenza di una famiglia di funzionali ellittici. Boll. Un. Mat. Ital. A 14 (1977) $526-529$.

[34] L. Modica and S. Mortola, Un esempio di $\Gamma^{-}$-convergenza. Boll. Un. Mat. Ital. B 14 (1977) 285-299.

[35] F. Morgan, Size-minimizing rectifiable currents. Invent. Math. 96 (1989) 333-348.

[36] S. Müller, Det = det. A remark on the distributional determinant. C. R. Acad. Sci. Paris Sér. I Math. 311 (1990) 13-17.

[37] S. Müller and S.J. Spector, An existence theory for nonlinear elasticity that allows for cavitation. Arch. Rational Mech. Anal. 131 (1995) 1-66.

[38] D. Mumford and J. Shah, Optimal approximations by piecewise smooth functions and associated variational problems. Comm. Pure Appl. Math. 42 (1989) 577-685.

[39] E. Sandier and S. Serfaty, Vortices in the magnetic Ginzburg-Landau model, vol. 70 of Progress Non. Differ. Eqs. Appl. Birkhäuser Boston Inc., Boston, MA (2007).

[40] V. Šverák, Regularity properties of deformations with finite energy. Arch. Rational Mech. Anal. 100 (1988) $105-127$.

[41] G. Talenti. Best constant in Sobolev inequality. Ann. Mat. Pura Appl. 110 (1976) 353-372.

[42] B. White, Rectifiability of flat chains. Ann. Math. 150 (1999) 165-184.

[43] W.P. Ziemer, Weakly differentiable functions, Sobolev spaces and functions of bounded variation, vol. 120 of Graduate Texts in Mathematics. Springer-Verlag, New York (1989). 